\title{
Dyslipidemia and Cardiovascular Disease Risk Factor Management in HIV-1-Infected Subjects Treated with HAART in the Spanish VACH Cohort
}

\author{
Pere Domingo ${ }^{*}, 1$, Ignacio Suarez-Lozano ${ }^{2}$, Ramón Teira ${ }^{3}$, Fernando Lozano ${ }^{4}$, Alberto Terrón ${ }^{5}$, \\ Pompeyo Viciana ${ }^{6}$, Juan González ${ }^{7}, \mathrm{M}^{\mathrm{a}}$ José Galindo $^{8}$, Paloma Geijo ${ }^{9}$, Antonio Vergara ${ }^{10}$, \\ Jaime Cosín ${ }^{11}$, Esteban Ribera ${ }^{12}$, Bernardino Roca ${ }^{13}, \mathrm{M}^{\mathrm{a}}$ Luisa Garcia-Alcalde ${ }^{14}$, \\ Trinitario Sánchez ${ }^{15}$, Ferran Torres ${ }^{16}$, Juan Ramón Lacalle ${ }^{17}$ and \\ Myriam Garrido ${ }^{18}$
}

Hospital de la Santa Creu i Sant Pau ${ }^{1}$, H. I. Elena-Huelva ${ }^{2}$, H. de Basurto-Bilbao ${ }^{3}$, H. de Valme-Sevilla, H. Clinico Puerto Real ${ }^{5}$, H. V. del Rocío-Sevilla ${ }^{6}$, H. La Paz-Madrid ${ }^{7}, H$. Clinico-Valencia ${ }^{8}, H$. V de la Luz-Cuenca ${ }^{9}$, H. SAS-Jerez ${ }^{10}, H$. Gregorio Marañon-Madrid ${ }^{11}$, H. Vall D'hebron-Barcelona ${ }^{12}$, H. General de Castellon ${ }^{13}$, H. de Cabueñes-Asturias ${ }^{14}, H$. V. del Rosell-Cartagena ${ }^{15}$, Dpto. de Estadística, Facultad de Medicina, Universitat Autònoma de Barcelona ${ }^{16}$, Dpto. Estadística, Facultad de Medicina Universidad Sevilla ${ }^{17}$, Data Management VACH Group ${ }^{18}$

\begin{abstract}
Background: There is increasing evidence that metabolic adverse effects associated with antiretroviral therapy may translate into an increased cardiovascular risk in HIV-1-infected patients.

Objectives: To determine the prevalence of risk factors for cardiovascular disease (CVD) among HIV-1-infected persons, and to investigate any association between them, stage of HIV-1 disease, and use of antiretroviral therapies.

Methods: Multicentric, cross-sectional analysis of CVD risk factors of treated patients in the VACH cohort. The data collected includes: demographic variables, cigarette smoking, diabetes mellitus, hypertension, dyslipidemia, body mass index, stage of HIV-1 infection, and antiretroviral therapy.

Results: The analysis included 2358 patients. More than $18 \%$ of the study population was at an age of appreciable risk of CVD. $1.7 \%$ had previous CVD and 59.2\% were smokers. Increased prevalence of elevated total cholesterol was observed among subjects receiving an NNRTI but no PI [odds ratio (OR), 3.34; 95\% confidence interval (CI), 1.77-6.31], PI but no NNRTI (OR, 4.04; 95\% CI, 2.12-7.71), or NNRTI + PI (OR, 17.77; 95\% CI, 7.24-43.59) compared to patients treated only with nucleoside reverse transcriptase inhibitors (NRTI). Higher CD4 cell count, lower plasma HIV-1 RNA levels, clinical signs of lipodystrophy, longer exposure times to NNRTI and PI, and older age were all also associated with elevated cholesterol levels. The use of lipid lowering agents was very low among our patients.

Conclusion: Patients in the VACH cohort present multiple known risk factors for CVD, and a very low rate of lipid lowering therapy use. NNRTI and/or PI-based antiretroviral therapies are associated with the worst lipid profile. This is more frequent in older subjects with greater CD4 counts and controlled HIV-1 replication.
\end{abstract}

Keywords: HIV-1 infection, antiretroviral therapy, HIV-1 protease inhibitors, non nucleoside reverse transcriptase inhibitors, dyslipidemia, cardiovascular risk, coronary heart disease, VACH.

\section{INTRODUCTION}

The widespread use of highly active antiretroviral therapy (HAART) has converted HIV-1 infection into a chronic manageable illness that needs lifelong therapy. However, HAART is associated with metabolic side effects including hypercholesterolemia, hypertriglyceridemia, insulin resistance and more rarely diabetes mellitus, and possibly arterial hypertension [1-4]. HAART-associated dyslipidemia is associated with accelerated atherosclerosis [5-7] and signs of endothelial dysfunction [8]. How all these facts are

\footnotetext{
*Address correspondence to this author at the Infectious Diseases Unit, Hospital de la Santa Creu I Sant Pau, Av. Sant Antoni Ma Claret, 167, 08025, Barcelona, Spain; Tel: +34935565609; Fax: + 34935565938;

E-mail: pdomingo@santpau.es or pere.domingo@uab.es
}

translated into clinical events of cardiovascular disease on a population level has been examined by a number of studies including cohort studies from the French Hospital Database [9], the HOPS Cohort [10], and the D:A:D multicohort study [11-13]. Overall, data from these cohorts suggest an increased risk of coronary heart disease (CHD) for HIV-1infected patients on HAART [9-13].

To assess the risk of treatment-associated cardiovascular risk factors, we performed a cross-sectional analysis of the HIV-1-infected patients included in the Spanish VACH Cohort. The objectives of the present analysis were to determine the proportion of patients with an increased risk profile for cardiovascular disease. Furthermore, we tried to identify factors associated with the increased risk profile. Addition- 
ally, our research was focused on the management of dyslipidemia in our cohort.

\section{PATIENTS AND METHODS}

\section{Design}

The study is a multi-centre, cross-sectional study of the prevalence and management of dyslipidemia and other established CHD/CVD risk factors in all HIV-1-infected subjects, aged 18 or above, treated with HAART, in routine clinical practice from the Spanish VACH cohort treatment centers. Characteristics of VACH Cohort have been described elsewhere [14].

\section{Study Population}

Male or female subjects aged 18 years or above at the time of enrollment with a documented HIV-1 infection, which attend VACH cohort outpatient HIV-1 treatment centers for routine, scheduled, clinical appointments, were eligible for this study.

In order to be eligible, subjects must have been on at least three antiretroviral drugs, at the time of the study visit. Antiretroviral (ARV) naïve subjects or ARV experienced, but currently untreated subjects or those currently treated with NRTI bi- or mono therapy were not eligible for this study. However, for the purpose of the study, we included patients treated with 3 NRTIs. Subjects who were hospitalized or have a frank cognitive impairment such as delirium or dementia on enrolment were not eligible either. Informed consent was obtained from the patients at study entry

\section{Data Collection}

In the VACH Cohort, data are prospectively collected according to standardized criteria, and are electronically stored in the Aplicación de Control Hospitalario $\left(\mathrm{AC} \& \mathrm{H}^{\mathrm{TM}}\right)$, an application specifically developed for the management of the cohort data. On enrolment, standardized data collection electronic forms were completed at the sites providing information from physical examination, patient interview and patient case notes, concerning family history of coronary heart disease, patients' prior history of CVD and diabetes, cigarette smoking, blood pressure, therapy for diabetes mellitus, lipidlowering and anti-hypertensive therapy, the presence of clinical signs of lipodystrophy and fasting serum lipid levels. Further, all cumulative data characterizing the patient's underlying HIV-1 infection since inclusion in any of the individual cohorts were collected, including information on demography, antiretroviral therapy, CD4 cell counts and HIV-1 viral loads. Dates of diagnosis of all AIDS-defining diseases are recorded, using the 1993 clinical definition of AIDS from the Centers for Disease Control and Prevention [15].

\section{VARIABLES}

\section{HIV-1 Laboratory Parameters}

CD4 cell count was stratified in strata of $100 \times 10^{6}$ (cells/l) or assessed as a continuous variable ( $\log _{2}$ transformed). Similarly, HIV-1 RNA was stratified in strata of: $<500,501-10$
000, $10001-100000$, and $>100000$ copies $/ \mathrm{ml}$, and also assessed as a continuous variable ( $\log _{10}$ transformed).

\section{Antiretroviral Therapy}

Four categories were predefined: (i) currently receiving only NRTI; (ii) currently receiving NNRTI and NRTI but not PI; (iii) currently receiving PI and NRTI but not NNRTI; or (iv) currently receiving PI, NNRTI and NRTI. Previous antiretroviral therapy exposure was modelled as cumulative time spent using each of the three drug classes.

\section{Cardiovascular Risk Factors and Managing Therapies}

The grouping of the risk factors assessed was defined prior to the initiation of the analysis. CVD risk factors were assessed as dichotomous categorical variables, where the cut-off levels chosen were conservative estimates of 'high risk' based on levels used for risk scoring in the background population [16-19]. Patients were considered to be at high risk for CVD if they had dad a prior CVD event or had diabetes or more than two risk factors accounting for a CVD 10 yr risk $>20 \%$. Moderate-high risk was considered if patients had more than two risks factors accounting for a CVD $10 \mathrm{yr}$ risk of $10-20 \%$, whereas moderate risk was considered when patients more than 2 risk factors and a CVD $10 \mathrm{yr}$ risk of less than $10 \%$. Finally low risk patients were those who had no or only a risk factor.

\section{Cardiovascular Risk Calculation}

Ten-year CHD risk estimates were calculated in accordance with the equations of Wilson et al. [16] with use of sex-specific risk calculations based on age, total and HDL cholesterol levels, systolic and diastolic blood pressure, presence of diabetes (defined as a fasting glucose level of $140 \mathrm{mg} / \mathrm{dL}$ ), family history of CHD, personal history of $\mathrm{CHD}$, and smoking status. The equation estimates the 10year risk for CHD events, including angina pectoris, myocardial infarction, and death due to CHD.

\section{Statistical Analyses}

We used frequencies, percentages and its $95 \%$ CI to describe categorical data, and median with interquartile range (IQR) for continuous variables. Univariable analysis was performed by means of the chi-squared and the KruskalWallis tests to compare categorical and continuous baseline demographic, clinical and laboratory characteristics. Association of CVD risk factors with antiretroviral therapy, demographic, clinical and laboratory parameters were tested in univariable logistic regression models. Multivariable logistic regression was then performed to identify parameters independently associated with the presence of CVD risk factors. The multivariable model included all parameters significantly associated with the risk factor assessed, at a level of $\mathrm{p}<0.05$ in the univariable model.

The analysis was performed using SAS version 9.1.3 software (SAS Institute Inc, Cary, North Carolina, USA) and the level of significance was established at the 0.05 level (two-sided). 


\section{RESULTS}

\section{Demographics}

By April 2004, the central database contained information on 2358 patients enrolled in VACH from fifteen participating Hospital cohorts. The patient characteristics are shown in Table $\mathbf{1 .}$

\section{Antiretroviral Therapy}

On enrolment, 9.9\% were receiving a regimen containing NRTI only, $46.4 \%$ were receiving NNRTI-based therapy, $41.9 \%$ PI-based therapy and $1.7 \%$ was on a regimen containing all three drug classes (Table 1). Sixty four percent of patients taking PI-based therapy had ritonavir-boosted PI on board while $0.02 \%$ were taking atazanavir. Overall, $72.6 \%$ of

Table 1. Demographic, Laboratory, Clinical and Antiretroviral Therapy Characteristics of the VACH Population at Baseline According to Current Usage of ART Drug Classes

\begin{tabular}{|c|c|c|c|c|c|c|}
\hline $\begin{array}{l}\text { Current ART } \\
\text { at Enrolment }\end{array}$ & $\begin{array}{l}\text { NRTI Only } \\
(\mathrm{n}=\mathbf{2 3 3})\end{array}$ & $\begin{array}{l}\text { Combination with } \\
\text { NNRTI }(n=1094)\end{array}$ & $\begin{array}{l}\text { Combination with } \\
\text { PI }(\mathbf{n}=990)\end{array}$ & $\begin{array}{c}\text { Combination with PI Plus } \\
\text { NNRTI }(n=41)\end{array}$ & $\begin{array}{c}\text { Total } \\
(\mathbf{n}=\mathbf{2 3 5 8})\end{array}$ & $\mathbf{p}$ \\
\hline $\begin{array}{l}\text { Age (years), } \\
\text { median [IQR] }\end{array}$ & $\begin{array}{c}39.00 \\
{[35.00,43.00]}\end{array}$ & $\begin{array}{c}40.00 \\
{[35.00,45.00]}\end{array}$ & $\begin{array}{c}39.00 \\
{[36.00,43.00]}\end{array}$ & $\begin{array}{c}41.00 \\
{[38.00,44.00]}\end{array}$ & $\begin{array}{c}40.00 \\
{[35.00,44.00]}\end{array}$ & 0.0852 \\
\hline $\begin{array}{l}\text { Sex }(\% \text { female }) \\
{[95 \% \mathrm{CI}]}\end{array}$ & $\begin{array}{c}54(23.2 \%) \\
{[17.9 \%, 29.1 \%]}\end{array}$ & $\begin{array}{c}243(22.2 \%) \\
{[19.8 \%, 24.8 \%]}\end{array}$ & $\begin{array}{c}217(21.9 \%) \\
{[19.4 \%, 24.6 \%]}\end{array}$ & $\begin{array}{c}8(19.5 \%) \\
{[8.8 \%, 34.9 \%]}\end{array}$ & $\begin{array}{c}522(22.1 \%) \\
{[20.5 \%, 23.9 \%]}\end{array}$ & 0.9450 \\
\hline $\begin{array}{l}\text { AIDS (\%) } \\
{[95 \% \mathrm{CI}]}\end{array}$ & $\begin{array}{c}57(24.5 \%) \\
{[19.1 \%, 30.5 \%]}\end{array}$ & $\begin{array}{c}346(31.6 \%) \\
{[28.9 \%, 34.5 \%]}\end{array}$ & $\begin{array}{c}367(37.1 \%) \\
{[34.1 \%, 40.2 \%]}\end{array}$ & $18(43.9 \%)[28.5 \%, 60.3 \%]$ & $\begin{array}{c}788(33.4 \%) \\
{[31.5 \%, 35.4 \%]}\end{array}$ & 0.0005 \\
\hline \multicolumn{6}{|l|}{ HIV Acquisition ${ }^{\&}$} & $<0.0001$ \\
\hline Injecting drug use & $\begin{array}{c}121(52.2 \%) \\
{[45.5 \%, 58.7 \%]}\end{array}$ & $\begin{array}{c}443(40.8 \%) \\
{[37.9 \%, 43.8 \%]}\end{array}$ & $\begin{array}{c}558(56.7 \%) \\
{[53.5 \%, 59.8 \%]}\end{array}$ & $\begin{array}{c}18(43.9 \%) \\
{[28.5 \%, 60.3 \%]}\end{array}$ & $\begin{array}{c}1140(48.7 \%) \\
{[46.6 \%, 50.7 \%]}\end{array}$ & \\
\hline Homosexual & $\begin{array}{c}32(13.8 \%) \\
{[9.6 \%, 18.9 \%]}\end{array}$ & $\begin{array}{c}245(22.6 \%) \\
{[20.1 \%, 25.2 \%]}\end{array}$ & $\begin{array}{c}137(13.9 \%) \\
{[11.8 \%, 16.2 \%]}\end{array}$ & $\begin{array}{c}8(19.5 \%) \\
{[8.8 \%, 34.9 \%]}\end{array}$ & $\begin{array}{c}422(18.0 \%) \\
{[16.5 \%, 19.6 \%]}\end{array}$ & \\
\hline Heterosexual & $\begin{array}{c}73(31.5 \%) \\
{[25.5 \%, 37.9 \%]}\end{array}$ & $\begin{array}{c}344(31.7 \%) \\
{[28.9 \%, 34.5 \%]}\end{array}$ & $\begin{array}{c}240(24.4 \%) \\
{[21.7 \%, 27.2 \%]}\end{array}$ & $\begin{array}{c}10(24.4 \%) \\
{[12.4 \%, 40.3 \%]}\end{array}$ & $\begin{array}{c}667(28.5 \%) \\
{[26.6 \%, 30.3 \%]}\end{array}$ & \\
\hline Others & $\begin{array}{c}6(2.6 \%) \\
{[1.0 \%, 5.5 \%]}\end{array}$ & $\begin{array}{c}54(5.0 \%) \\
{[3.8 \%, 6.4 \%]}\end{array}$ & $\begin{array}{c}49(5.0 \%) \\
{[3.7 \%, 6.5 \%]}\end{array}$ & $\begin{array}{c}5(12.2 \%) \\
{[4.1 \%, 26.2 \%]}\end{array}$ & $\begin{array}{c}114(4.9 \%) \\
{[4.0 \%, 5.8 \%]}\end{array}$ & \\
\hline $\begin{array}{l}\text { CD4 cell count (x 106/l), } \\
\text { median [IQR] \& }\end{array}$ & $\begin{array}{c}510.00 \\
{[358.00,735.00]}\end{array}$ & $\begin{array}{c}459.00 \\
{[288.00,688.00]}\end{array}$ & $\begin{array}{c}368.00 \\
{[219.50,568.50]}\end{array}$ & $\begin{array}{c}427.00 \\
{[251.00,756.00]}\end{array}$ & $\begin{array}{c}426.00 \\
{[256.00,651.00]}\end{array}$ & $<0.0001$ \\
\hline $\begin{array}{l}\text { HIV-1 RNA }\left(\log _{10}\right) \text {, } \\
\text { median [IQR] }\end{array}$ & $\begin{array}{c}1.69 \\
{[1.69,1.70]}\end{array}$ & $\begin{array}{c}1.69 \\
{[1.69,1.99]}\end{array}$ & $\begin{array}{c}1.70 \\
{[1.69,2.44]}\end{array}$ & $\begin{array}{c}1.70 \\
{[1.69,2.30]}\end{array}$ & $\begin{array}{c}1.69 \\
{[1.69,2.17]}\end{array}$ & $<0.0001$ \\
\hline $\begin{array}{l}\text { HIV-1 RNA copies/mL, } \\
\text { median [IQR }]^{\&}\end{array}$ & $\begin{array}{c}49.00 \\
{[49.00,50.00]}\end{array}$ & $\begin{array}{c}49.00 \\
{[49.00,97.00]}\end{array}$ & $\begin{array}{c}50.00 \\
{[49.00,275.00]}\end{array}$ & $\begin{array}{c}50.00 \\
{[49.00,200.00]}\end{array}$ & $\begin{array}{c}49.00 \\
{[49.00,148.00]}\end{array}$ & 0.0010 \\
\hline $\begin{array}{l}\text { HIV-1 RNA }\left(\log _{10}\right) \\
\text { 0median [IQR] }\end{array}$ & $\begin{array}{c}1.69 \\
{[1.69,1.70]}\end{array}$ & $\begin{array}{c}1.69 \\
{[1.69,1.99]}\end{array}$ & $\begin{array}{c}1.70 \\
{[1.69,2.44]}\end{array}$ & $\begin{array}{c}1.70 \\
{[1.69,2.30]}\end{array}$ & $\begin{array}{c}1.69 \\
{[1.69,2.17]}\end{array}$ & $<0.0001$ \\
\hline $\begin{array}{l}\text { ART exposure } \\
{[\text { median (IQR)] }}\end{array}$ & $\begin{array}{c}4.68 \\
{[2.88,6.27]}\end{array}$ & $\begin{array}{c}4.12 \\
{[2.01,6.01]}\end{array}$ & $\begin{array}{c}4.31 \\
{[1.73,6.22]}\end{array}$ & $\begin{array}{c}6.33 \\
{[4.32,8.94]}\end{array}$ & $\begin{array}{c}4.29 \\
{[1.98,6.19]}\end{array}$ & $<0.0001$ \\
\hline $\begin{array}{l}\text { HAART exposure } \\
\text { [median (IQR)] }\end{array}$ & $\begin{array}{c}4.59 \\
{[2.71,5.96]}\end{array}$ & $\begin{array}{c}4.00 \\
{[1.93,5.61]}\end{array}$ & $\begin{array}{c}4.04 \\
{[1.64,5.80]}\end{array}$ & $\begin{array}{c}6.11 \\
{[4.10,6.95]}\end{array}$ & $\begin{array}{c}4.10 \\
{[1.90,5.79]}\end{array}$ & $<0.0001$ \\
\hline $\begin{array}{l}\text { Exposure to PI (\%) } \\
{[95 \% \mathrm{CI}]}\end{array}$ & $\begin{array}{c}153(65.7 \%) \\
{[59.2 \%, 71.7 \%]}\end{array}$ & $\begin{array}{c}527(48.2 \%) \\
{[45.2 \%, 51.2 \%]}\end{array}$ & $\begin{array}{c}990(100.0 \%) \\
{[99.7 \%, 100.0 \%]}\end{array}$ & $\begin{array}{c}41(100.0 \%) \\
{[93.0 \%, 100.0 \%]}\end{array}$ & $\begin{array}{c}1711(72.6 \%) \\
{[70.7 \%, 74.4 \%]}\end{array}$ & $<0.0001$ \\
\hline $\begin{array}{l}\text { Duration (years), } \\
\text { median [IQR] }\end{array}$ & $\begin{array}{c}4.27 \\
{[0.00,5.95]}\end{array}$ & $\begin{array}{c}0.00 \\
{[0.00,5.55]}\end{array}$ & $\begin{array}{c}3.80 \\
{[1.21,5.86]}\end{array}$ & $\begin{array}{c}6.11 \\
{[2.53,6.98]}\end{array}$ & $\begin{array}{c}3.26 \\
{[0.00,5.80]}\end{array}$ & $<0.0001$ \\
\hline $\begin{array}{l}\text { Exposure to NNRTIs (\%) } \\
{[95 \% \mathrm{CI}]}\end{array}$ & $\begin{array}{c}88(37.8 \%) \\
{[31.5 \%, 44.3 \%]}\end{array}$ & $\begin{array}{c}1094(100.0 \%) \\
{[99.7 \%, 100.0 \%]}\end{array}$ & $\begin{array}{c}357(36.1 \%) \\
{[33.1 \%, 39.1 \%]}\end{array}$ & $\begin{array}{c}41(100.0 \%) \\
{[93.0 \%, 100.0 \%]}\end{array}$ & $\begin{array}{c}1580(67.0 \%) \\
{[65.1 \%, 68.9 \%]}\end{array}$ & $<0.0001$ \\
\hline $\begin{array}{l}\text { Duration (years), } \\
\text { median [IQR] }\end{array}$ & $\begin{array}{c}0.00 \\
{[0.00,2.80]}\end{array}$ & $\begin{array}{c}2.69 \\
{[1.19,4.08]}\end{array}$ & $\begin{array}{c}0.00 \\
{[0.00,2.32]}\end{array}$ & $\begin{array}{c}3.16 \\
{[1.67,4.26]}\end{array}$ & $\begin{array}{c}1.51 \\
{[0.00,3.64]}\end{array}$ & $<0.0001$ \\
\hline $\begin{array}{l}\text { Exposure to NRTI (\%) } \\
{[95 \% \mathrm{CI}]}\end{array}$ & $\begin{array}{c}233(100.0 \%) \\
{[98.7 \%, 100.0 \%]}\end{array}$ & $\begin{array}{c}1092(99.8 \%) \\
{[99.3 \%, 100.0 \%]}\end{array}$ & $\begin{array}{c}990(100.0 \%) \\
{[99.7 \%, 100.0 \%]}\end{array}$ & $\begin{array}{c}40(97.6 \%) \\
{[87.1 \%, 99.9 \%]}\end{array}$ & $\begin{array}{c}2355(99.9 \%) \\
{[99.6 \%, 100.0 \%]}\end{array}$ & 0.0003 \\
\hline $\begin{array}{l}\text { Duration (years), } \\
\text { median [IQR] }\end{array}$ & $\begin{array}{c}4.68 \\
{[2.88,6.27]}\end{array}$ & $\begin{array}{c}4.10 \\
{[2.01,6.01]}\end{array}$ & $\begin{array}{c}4.31 \\
{[1.72,6.22]}\end{array}$ & $\begin{array}{c}6.33 \\
{[4.32,8.94]}\end{array}$ & $\begin{array}{c}4.28 \\
{[1.98,6.19]}\end{array}$ & $<0.0001$ \\
\hline
\end{tabular}

Descriptive statistics are n (\%) [95\%CI] for qualitative, and median [IQR] for qualitative variables. NRTI = nucleoside reverse transcriptase inhibitors, NNRTI = non-nucleoside reverse transcriptase inhibitors, $\mathrm{PI}=$ protease inhibitors, AIDS = acquired immunodeficiency syndrome, ART $=$ antiretroviral therapy, HAART $=$ highly active antiretroviral therapy. ${ }^{\&}$ Percentage of missingness $<1 \%$; null percentage for the rest of variables. 
the study population had at any time been exposed to PI with a median exposure time of 3.3 years (IQR, $0-5.8$ years), $67.0 \%$ had been exposed to NNRTI with a median exposure time of 1.5 years (IQR, $0-3.6$ years) and $99.9 \%$ had been exposed to NRTI with a median exposure of 4.3 years (IQR, 2.0-6.2 years) (Table $\mathbf{1}$ ).

\section{CVD Risk Factors and Association with Antiretroviral Therapy}

CVD risk factors were prevalent in the study population (Table 2). More than $18 \%$ of the study population was in an age group constituting a CVD risk factor. Thirteen percent had a family history of coronary heart disease, and $1.7 \%$ had a previous history of CVD. Almost $60 \%$ of the study population was current cigarette smokers.

\section{Serum Total Cholesterol}

The association of antiretroviral therapy with lipid levels is shown in Table 2. Assessed from median cholesterol levels (Table 2) and in univariable models (Table 3), patients currently using regimens containing all three drug classes were at increased risk of having a high total cholesterol when

Table 2. Cardiovascular Risk Factors in the VACH Cohort Population According to Current Usage of Antiretroviral Therapy (ART) Drug Classes

\begin{tabular}{|c|c|c|c|c|c|c|}
\hline & $\begin{array}{l}\text { NRTI Only } \\
(\mathbf{n}=\mathbf{2 3 3})\end{array}$ & $\begin{array}{l}\text { Combination with } \\
\text { NNRTI }(n=1094)\end{array}$ & $\begin{array}{l}\text { Combination with } \\
\text { PI }(n=990)\end{array}$ & $\begin{array}{l}\text { Combination with PI } \\
\text { Plus NNRTI }(n=41)\end{array}$ & $\begin{array}{c}\text { Total } \\
(\mathrm{n}=\mathbf{2 3 5 8})\end{array}$ & $\mathbf{p}$ \\
\hline $\begin{array}{l}\text { Age }>45 \text { years male, } \\
>55 \text { female, } \\
(\%)[95 \% \mathrm{CI}]^{\&}\end{array}$ & $\begin{array}{c}33(14.2 \%) \\
{[10.0 \%, 19.3 \%]}\end{array}$ & $\begin{array}{c}244(22.3 \%) \\
{[19.9 \%, 24.9 \%]}\end{array}$ & $\begin{array}{c}142(14.4 \%) \\
{[12.2 \%, 16.7 \%]}\end{array}$ & $\begin{array}{c}7(17.1 \%) \\
{[7.2 \%, 32.1 \%]}\end{array}$ & $\begin{array}{c}426(18.1 \%) \\
{[16.5 \%, 19.7 \%]}\end{array}$ & 0.0001 \\
\hline $\begin{array}{l}\text { Body mass index, } \\
\text { median [IQR] }\end{array}$ & $\begin{array}{c}24.10 \\
{[22.28,26.22]}\end{array}$ & $\begin{array}{c}23.82 \\
{[21.81,25.59]}\end{array}$ & $\begin{array}{c}23.56 \\
{[21.52,25.56]}\end{array}$ & $\begin{array}{c}23.56 \\
{[21.05,25.95]}\end{array}$ & $\begin{array}{c}23.82 \\
{[21.75,25.67]}\end{array}$ & 0.0700 \\
\hline $\begin{array}{l}\text { Body mass index }>30 \mathrm{~kg} / \mathrm{m}^{2} \text {, } \\
(\%)[95 \% \mathrm{CI}]\end{array}$ & $\begin{array}{c}9(3.9 \%) \\
{[1.8 \%, 7.2 \%]}\end{array}$ & $\begin{array}{c}29(2.7 \%) \\
{[1.8 \%, 3.8 \%]}\end{array}$ & $\begin{array}{c}41(4.1 \%) \\
{[3.0 \%, 5.6 \%]}\end{array}$ & $\begin{array}{c}3(7.3 \%) \\
{[1.5 \%, 19.9 \%]}\end{array}$ & $\begin{array}{c}82(3.5 \%) \\
{[2.8 \%, 4.3 \%]}\end{array}$ & 0.1428 \\
\hline $\begin{array}{l}\text { Current smoker, } \\
(\%)[95 \% \mathrm{CI}]\end{array}$ & $\begin{array}{c}146(62.7 \%) \\
{[56.1 \%, 68.9 \%]}\end{array}$ & $\begin{array}{c}588(53.7 \%) \\
{[50.7 \%, 56.7 \%]}\end{array}$ & $\begin{array}{c}641(64.7 \%) \\
{[61.7 \%, 67.7 \%]}\end{array}$ & $\begin{array}{c}22(53.7 \%) \\
{[37.4 \%, 69.3 \%]}\end{array}$ & $\begin{array}{c}1397(59.2 \%) \\
{[57.2 \%, 61.2 \%]}\end{array}$ & $<0.0001$ \\
\hline $\begin{array}{l}\text { Family story of CVD, } \\
(\%)[95 \% \mathrm{CI}]\end{array}$ & $\begin{array}{c}30(12.9 \%) \\
{[8.9 \%, 17.9 \%]}\end{array}$ & $\begin{array}{c}157(14.4 \%) \\
{[12.3 \%, 16.6 \%]}\end{array}$ & $\begin{array}{c}116(11.7 \%) \\
{[9.8 \%, 13.9 \%]}\end{array}$ & $\begin{array}{c}3(7.3 \%) \\
{[1.5 \%, 19.9 \%]}\end{array}$ & $\begin{array}{c}306(13.0 \%) \\
{[11.6 \%, 14.4 \%]}\end{array}$ & 0.2227 \\
\hline $\begin{array}{l}\text { Previous CVD, } \\
\text { (\%) }[95 \% \mathrm{CI}]\end{array}$ & $\begin{array}{c}7(3.0 \%) \\
{[1.2 \%, 6.1 \%]}\end{array}$ & $\begin{array}{c}17(1.6 \%) \\
{[0.9 \%, 2.5 \%]}\end{array}$ & $\begin{array}{c}16(1.6 \%) \\
{[0.9 \%, 2.6 \%]}\end{array}$ & $\begin{array}{c}0(0.0 \%) \\
{[0.0 \%, 7.0 \%]}\end{array}$ & $\begin{array}{c}40(1.7 \%) \\
{[1.2 \%, 2.3 \%]}\end{array}$ & 0.3520 \\
\hline $\begin{array}{l}\text { Hypertension, } \\
(\%)[95 \% \mathrm{CI}]^{\text {\& }}\end{array}$ & $\begin{array}{c}50(21.5 \%) \\
{[16.4 \%, 27.3 \%]}\end{array}$ & $\begin{array}{c}272(24.9 \%) \\
{[22.3 \%, 27.5 \%]}\end{array}$ & $\begin{array}{c}212(21.4 \%) \\
{[18.9 \%, 24.1 \%]}\end{array}$ & $\begin{array}{c}5(12.2 \%) \\
{[4.1 \%, 26.2 \%]}\end{array}$ & $\begin{array}{c}539(22.9 \%) \\
{[21.2 \%, 24.6 \%]}\end{array}$ & 0.0886 \\
\hline $\begin{array}{l}\text { Diabetes mellitus, } \\
(\%)[95 \% \mathrm{CI}]\end{array}$ & $\begin{array}{c}21(9.0 \%) \\
{[5.7 \%, 13.4 \%]}\end{array}$ & $\begin{array}{c}98(9.0 \%) \\
{[7.3 \%, 10.8 \%]}\end{array}$ & $\begin{array}{c}52(5.3 \%) \\
{[3.9 \%, 6.8 \%]}\end{array}$ & $\begin{array}{c}1(2.4 \%) \\
{[0.1 \%, 12.9 \%]}\end{array}$ & $\begin{array}{l}172(7.3 \%) \\
{[6.3 \%, 8.4 \%]}\end{array}$ & 0.0046 \\
\hline $\begin{array}{l}\text { Total cholesterol (mmol/1), } \\
\text { median [IQR] }\end{array}$ & $\begin{array}{c}4.56 \\
{[3.83,5.13]}\end{array}$ & $\begin{array}{c}4.97 \\
{[4.20,5.78]}\end{array}$ & $\begin{array}{c}4.82 \\
{[4.12,5.65]}\end{array}$ & $\begin{array}{c}5.85 \\
{[4.61,6.94]}\end{array}$ & $\begin{array}{c}4.87 \\
{[4.12,5.67]}\end{array}$ & $<0.0001$ \\
\hline $\begin{array}{l}\text { Total cholesterol }>6.2 \mathrm{mmol} / 1 \text {, } \\
(\%)[95 \% \mathrm{CI}]\end{array}$ & $\begin{array}{c}11(4.7 \%) \\
{[2.4 \%, 8.3 \%]}\end{array}$ & $\begin{array}{c}172(15.7 \%) \\
{[13.6 \%, 18.0 \%]}\end{array}$ & $\begin{array}{c}145(14.6 \%) \\
{[12.5 \%, 17.0 \%]}\end{array}$ & $\begin{array}{c}18(43.9 \%) \\
{[28.5 \%, 60.3 \%]}\end{array}$ & $\begin{array}{c}346(14.7 \%) \\
{[13.3 \%, 16.2 \%]}\end{array}$ & $<0.0001$ \\
\hline $\begin{array}{l}\text { HDL cholesterol }(\mathrm{mmol} / \mathrm{l}) \\
\text { median }[\mathrm{IQR}]\end{array}$ & $\begin{array}{c}1.09 \\
{[0.88,1.32]}\end{array}$ & $\begin{array}{c}1.27 \\
{[1.01,1.58]}\end{array}$ & $\begin{array}{c}1.17 \\
{[0.93,1.45]}\end{array}$ & $\begin{array}{c}1.19 \\
{[1.04,1.55]}\end{array}$ & $\begin{array}{c}1.19 \\
{[0.96,1.48]}\end{array}$ & $<0.0001$ \\
\hline $\begin{array}{l}\text { HDL cholesterol }<0.9 \mathrm{mmol} / 1 \text {, } \\
(\%)[95 \% \mathrm{CI}]\end{array}$ & $\begin{array}{c}60(25.8 \%) \\
{[20.3 \%, 31.9 \%]}\end{array}$ & $\begin{array}{c}157(14.4 \%) \\
{[12.3 \%, 16.6 \%]}\end{array}$ & $\begin{array}{c}216(21.8 \%) \\
{[19.3 \%, 24.5 \%]}\end{array}$ & $\begin{array}{c}6(14.6 \%) \\
{[5.6 \%, 29.2 \%]}\end{array}$ & $\begin{array}{c}439(18.6 \%) \\
{[17.1 \%, 20.2 \%]}\end{array}$ & $<0.0001$ \\
\hline $\mathrm{TC} / \mathrm{HDL}$ ratio & $\begin{array}{c}4.20 \\
{[3.13,5.19]}\end{array}$ & $\begin{array}{c}3.87 \\
{[3.07,4.95]}\end{array}$ & $\begin{array}{c}4.17 \\
{[3.18,5.30]}\end{array}$ & $\begin{array}{c}4.72 \\
{[3.68,5.77]}\end{array}$ & $\begin{array}{c}4.05 \\
{[3.13,5.13]}\end{array}$ & $<0.0001$ \\
\hline $\begin{array}{l}\text { LDL cholesterol }(\mathrm{mmol} / \mathrm{l}) \text {, } \\
\text { median }[\mathrm{IQR}]^{\&}\end{array}$ & $\begin{array}{c}2.51 \\
{[1.94,3.21]}\end{array}$ & $\begin{array}{c}2.90 \\
{[2.23,3.63]}\end{array}$ & $\begin{array}{c}2.75 \\
{[2.05,3.55]}\end{array}$ & $\begin{array}{c}3.44 \\
{[2.38,4.35]}\end{array}$ & $\begin{array}{c}2.81 \\
{[2.10,3.57]}\end{array}$ & $<0.0001$ \\
\hline $\begin{array}{l}\text { LDL cholesterol }>4.14 \mathrm{mmol} / 1 \text {, } \\
(\%)[95 \% \mathrm{CI}]^{\&}\end{array}$ & $\begin{array}{c}11(4.8 \%) \\
{[2.4 \%, 8.4 \%]}\end{array}$ & $\begin{array}{c}142(13.0 \%) \\
{[11.1 \%, 15.2 \%]}\end{array}$ & $\begin{array}{c}122(12.4 \%) \\
{[10.4 \%, 14.6 \%]}\end{array}$ & $\begin{array}{c}12(29.3 \%) \\
{[16.1 \%, 45.5 \%]}\end{array}$ & $\begin{array}{c}287(12.2 \%) \\
{[10.9 \%, 13.6 \%]}\end{array}$ & $<0.0001$ \\
\hline $\begin{array}{l}\text { Triglycerides (mmol/l), } \\
\text { median [IQR] }\end{array}$ & $\begin{array}{c}4.56 \\
{[3.83,5.13]}\end{array}$ & $\begin{array}{c}4.97 \\
{[4.20,5.78]}\end{array}$ & $\begin{array}{c}4.82 \\
{[4.12,5.65]}\end{array}$ & $\begin{array}{c}5.85 \\
{[4.61,6.94]}\end{array}$ & $\begin{array}{c}4.87 \\
{[4.12,5.67]}\end{array}$ & $<0.0001$ \\
\hline $\begin{array}{l}\text { Triglycerides }>2.3 \mathrm{mmol} / 1 \text {, } \\
(\%)[95 \% \mathrm{CI}]\end{array}$ & $\begin{array}{c}58(24.9 \%) \\
{[19.5 \%, 31.0 \%]}\end{array}$ & $\begin{array}{c}239(21.8 \%) \\
{[19.4 \%, 24.4 \%]}\end{array}$ & $\begin{array}{c}294(29.7 \%) \\
{[26.9 \%, 32.7 \%]}\end{array}$ & $\begin{array}{c}20(48.8 \%) \\
{[32.9 \%, 64.9 \%]}\end{array}$ & $\begin{array}{c}611(25.9 \%) \\
{[24.2 \%, 27.7 \%]}\end{array}$ & $<0.0001$ \\
\hline $\begin{array}{l}\text { Lipodystrophy, } \\
\text { (\%) [95\% CI] }\end{array}$ & $\begin{array}{c}41(17.6 \%) \\
{[12.9 \%, 23.1 \%]}\end{array}$ & $\begin{array}{c}200(18.3 \%) \\
{[16.0 \%, 20.7 \%]}\end{array}$ & $\begin{array}{c}168(17.0 \%) \\
{[14.7 \%, 19.5 \%]}\end{array}$ & $\begin{array}{c}11(26.8 \%) \\
{[14.2 \%, 42.9 \%]}\end{array}$ & $\begin{array}{c}420(17.8 \%) \\
{[16.3 \%, 19.4 \%]}\end{array}$ & 0.4027 \\
\hline
\end{tabular}

Descriptive statistics are $\mathrm{n}(\%)[95 \% \mathrm{CI}]$ for qualitative, and median $[\mathrm{IQR}]$ for qualitative variables. NRTI $=$ nucleoside reverse transcriptase inhibitors, NNRTI $=$ non-nucleoside reverse transcriptase inhibitors, $\mathrm{PI}=$ protease inhibitors, $\mathrm{CVD}=$ cardiovascular disease, $\mathrm{LDL}=$ low density lipoprotein, $\mathrm{HDL}=$ high density lipoprotein, $\mathrm{TC}=$ total cholesterol. ${ }^{\&}$ Percentage of missingness $<1 \%$; null percentage for the rest of variables. 
Table 3. Association of Current Antiretroviral Therapy (ART) with BMI, Hypertension, Diabetes, Dyslipidaemia, and Lipodystrophy at Baseline. Results from Logistic Regression Models, Univariable and Adjusted for Other Factors

\begin{tabular}{|c|c|c|c|c|c|c|c|c|c|c|c|c|c|}
\hline & $\begin{array}{c}\text { Only NRTI } \\
\text { Ref. }\end{array}$ & \multicolumn{4}{|c|}{ NNRTI } & \multicolumn{4}{|c|}{ PI } & \multicolumn{4}{|c|}{ NNRTI+PI } \\
\hline Univariant & [1] & 0.69 & 0.31 & 1.55 & 0.373 & 1.13 & 0.52 & 2.45 & 0.761 & 2.08 & 0.53 & 8.20 & 0.294 \\
\hline Multivariable & {$[1]$} & 0.63 & 0.28 & 1.42 & 0.266 & 1.13 & 0.52 & 2.46 & 0.756 & 1.91 & 0.48 & 7.57 & 0.359 \\
\hline Univariant & {$[1]$} & 1.12 & 0.66 & 1.90 & 0.672 & 0.59 & 0.34 & 1.04 & 0.069 & 0.28 & 0.04 & 2.15 & 0.221 \\
\hline Multivariable & {$[1]$} & 0.98 & 0.57 & 1.68 & 0.933 & 0.60 & 0.34 & 1.06 & 0.079 & 0.25 & 0.03 & 1.97 & 0.189 \\
\hline \multicolumn{14}{|c|}{$\mathrm{HDL}<=0.9 \mathrm{mmol} / \mathrm{l}$} \\
\hline Univariant & {$[1]$} & 0.48 & 0.34 & 0.68 & 0.000 & 0.79 & 0.56 & 1.11 & 0.174 & 0.49 & 0.19 & 1.22 & 0.124 \\
\hline Multivariable & {$[1]$} & 1.36 & 0.56 & 3.31 & 0.500 & 0.88 & 0.34 & 2.27 & 0.796 & NE & $\mathrm{NE}$ & NE & 0.983 \\
\hline \multicolumn{14}{|l|}{ Hypertension } \\
\hline Univariant & {$[1]$} & 1.35 & 0.94 & 1.94 & 0.105 & 1.10 & 0.76 & 1.59 & 0.599 & 0.57 & 0.21 & 1.53 & 0.265 \\
\hline Multivariable & [1] & 1.28 & 0.88 & 1.88 & 0.198 & 1.26 & 0.86 & 1.86 & 0.238 & 0.57 & 0.21 & 1.59 & 0.283 \\
\hline \multicolumn{14}{|l|}{ Lipodystrophy } \\
\hline Univariant & {$[1]$} & 0.99 & 0.68 & 1.44 & 0.970 & 0.92 & 0.63 & 1.35 & 0.685 & 1.59 & 0.74 & 3.44 & 0.237 \\
\hline Multivariable & [1] & 0.63 & 0.28 & 1.42 & 0.266 & 1.13 & 0.52 & 2.46 & 0.756 & 1.91 & 0.48 & 7.57 & 0.359 \\
\hline \multicolumn{14}{|c|}{ Cardiovascular Risk (moderate-high) } \\
\hline \multicolumn{14}{|c|}{$\mathrm{TG}>=2.3 \mathrm{mmol} / \mathrm{l}$} \\
\hline Univariant & {$[1]$} & 0.85 & 0.60 & 1.20 & 0.356 & 1.31 & 0.93 & 1.83 & 0.122 & 2.98 & 1.50 & 5.92 & 0.002 \\
\hline Multivariable & [1] & 0.81 & 0.57 & 1.15 & 0.241 & 1.40 & 0.99 & 1.99 & 0.059 & 2.80 & 1.39 & 5.65 & 0.004 \\
\hline
\end{tabular}

The final multivariable model included variables that were significantly $(\mathrm{P}<0.05)$ associated with the cardiovascular risk factor in question. The following variables were tested age, gender, smoking, family history of coronary heart disease, previous cardiovascular disease, body mass index, transmission mode, CD4 cell count, HIV RNA, previous AIDS. PI, protease inhibitor; NNRTI, non-nucleoside reverse transcriptase inhibitor; NRTI, nucleoside reverse transcriptase inhibitor; OR, odds ratio; CI, confidence interval; HDL, high density lipoprotein; TG, triglycerides.

compared with patients using regimen containing only NRTI. This pattern remained unchanged after controlling for other risk factors (Table 4). In a univariable logistic model for cumulative antiretroviral therapy exposure time, the OR increment of risk for elevated total cholesterol was $9 \%$ $(p=0.005), 2 \%(p=0.251)$ and $2 \%(p=0.314)$ per year of exposure to NRTI, NNRTI and PI, respectively (Table 3). The level of immunodeficiency and plasma HIV-1 RNA were independently associated with elevated total cholesterol after adjustment for other factors (Fig. 1). Overall, the adjusted risk of having elevated total cholesterol increased by $31 \%$ per twofold increase in CD4 cell count [OR, 1.31; IC95\%: 1.16-1.47 per $\left.\log _{2} C D 4, P<0.001\right]$ (Fig. 1). In all antiretrovi- ral therapy groups, higher HIV-1 viral load was associated with a decreased risk of elevated total cholesterol (Fig. 1); overall the adjusted OR was 0.77 (95\%CI: 0.68-0.88), $\mathrm{P}<$ 0.001 , per $1 \log _{10}$ increase in HIV-1 RNA.

\section{Serum Triglycerides}

In a univariable logistic model for cumulative antiretroviral drug exposure time, the OR for elevated triglycerides was 1.05 (95\%CI, 1.02-1.09), 1.02 (95\%CI, 0.97-1.07) and 1.06 (95\%CI, 1.02-1.09) per year of exposure to NRTI, NNRTI and PI, respectively, associations essentially unchanged in the multivariable model (Tables 3 and 4). Overall, the ad- 
Table 4. Risk for BMI, Hypertension, Diabetes, Dyslipidaemia, and Lipodystrophy at Baseline. Per Year of ART Exposure for HAART, NNRTI, NRTI, and PI. Results from Logistic Regression Models, Univariable and Adjusted for Other Factors

\begin{tabular}{|c|c|c|c|c|c|c|c|c|c|c|c|c|c|c|c|c|}
\hline & \multicolumn{4}{|c|}{$\begin{array}{l}\text { Risk Per Year of HAA } \\
\text { Exposure }\end{array}$} & \multicolumn{4}{|c|}{$\begin{array}{c}\text { Risk Per Year of NRTI } \\
\text { Exposure }\end{array}$} & \multicolumn{4}{|c|}{$\begin{array}{c}\text { Risk Per Year of NNRTI } \\
\text { Exposure }\end{array}$} & \multicolumn{4}{|c|}{$\begin{array}{l}\text { Risk Per Year of PI } \\
\text { Exposure }\end{array}$} \\
\hline & OR & \multicolumn{2}{|c|}{$95 \%$ CI } & $\mathbf{p}$ & OR & \multicolumn{2}{|c|}{$95 \% \mathrm{CI}$} & $\mathbf{p}$ & OR & \multicolumn{2}{|c|}{$95 \% \mathrm{CI}$} & $\mathbf{p}$ & OR & \multicolumn{2}{|c|}{$95 \% \mathrm{CI}$} & $\mathbf{p}$ \\
\hline \multicolumn{17}{|l|}{ BMI $>30 \mathrm{~kg} / \mathrm{m}^{2}$} \\
\hline Univariant & 0.95 & 0.86 & 1.05 & 0.329 & 0.93 & 0.85 & 1.01 & 0.091 & 0.96 & 0.85 & 1.09 & 0.546 & 0.98 & 0.90 & 1.06 & 0.570 \\
\hline Univariant & 1.09 & 1.01 & 1.17 & 0.018 & 1.06 & 1.00 & 1.12 & 0.036 & 1.11 & 1.02 & 1.20 & 0.011 & 1.03 & 0.97 & 1.09 & 0.295 \\
\hline Multivariable & 1.09 & 1.01 & 1.17 & 0.020 & 1.08 & 1.02 & 1.14 & 0.011 & 1.10 & 1.01 & 1.19 & 0.031 & 1.04 & 0.98 & 1.10 & 0.243 \\
\hline \multicolumn{17}{|c|}{ HDL $<=0.9 \mathrm{mmol} / \mathrm{l}$} \\
\hline Univariant & 0.99 & 0.94 & 1.03 & 0.590 & 1.00 & 0.96 & 1.03 & 0.834 & 0.93 & 0.88 & 0.99 & 0.018 & 1.01 & 0.97 & 1.05 & 0.627 \\
\hline \multicolumn{17}{|c|}{ Hypolipemiant drugs } \\
\hline Univariant & 1.18 & 1.06 & 1.32 & 0.003 & 1.11 & 1.02 & 1.20 & 0.014 & 1.22 & 1.08 & 1.39 & 0.002 & 1.08 & 0.99 & 1.18 & 0.097 \\
\hline Multivariable & 1.16 & 1.03 & 1.30 & 0.014 & 1.10 & 1.01 & 1.20 & 0.032 & 1.19 & 1.05 & 1.36 & 0.007 & 1.06 & 0.97 & 1.16 & 0.209 \\
\hline \multicolumn{17}{|l|}{ Hypertension } \\
\hline Univariant & 1.06 & 1.02 & 1.11 & 0.004 & 1.05 & 1.02 & 1.09 & 0.003 & 1.07 & 1.02 & 1.13 & 0.007 & 1.03 & 1.00 & 1.07 & 0.070 \\
\hline Multivariable & 1.06 & 1.01 & 1.11 & 0.017 & 1.06 & 1.02 & 1.10 & 0.002 & 1.06 & 1.00 & 1.11 & 0.051 & 1.03 & 1.00 & 1.07 & 0.070 \\
\hline \multicolumn{17}{|l|}{ Lipodystrophy } \\
\hline Univariant & 1.32 & 1.25 & 1.39 & 0.000 & 1.19 & 1.15 & 1.24 & 0.000 & 1.16 & 1.10 & 1.23 & 0.000 & 1.21 & 1.16 & 1.26 & 0.000 \\
\hline Multivariable & 1.30 & 1.23 & 1.37 & 0.000 & 1.18 & 1.14 & 1.23 & 0.000 & 1.13 & 1.07 & 1.20 & 0.000 & 1.19 & 1.14 & 1.24 & 0.000 \\
\hline \multicolumn{17}{|c|}{ Total Cholesterol $>=6.2 \mathrm{mmol} / \mathrm{l}$} \\
\hline Multivariable & 1.03 & 0.98 & 1.09 & 0.268 & 1.03 & 0.98 & 1.08 & 0.242 & 1.04 & 0.98 & 1.11 & 0.190 & 1.03 & 0.98 & 1.07 & 0.221 \\
\hline \multicolumn{17}{|c|}{$T G>=2.3 \mathrm{mmol} / \mathrm{l}$} \\
\hline Univariant & 1.06 & 1.02 & 1.11 & 0.003 & 1.05 & 1.02 & 1.09 & 0.001 & 1.02 & 0.97 & 1.07 & 0.370 & 1.06 & 1.02 & 1.09 & 0.001 \\
\hline Multivariable & 1.04 & 1.00 & 1.09 & 0.068 & 1.04 & 1.01 & 1.08 & 0.016 & 0.99 & 0.94 & 1.04 & 0.770 & 1.05 & 1.01 & 1.08 & 0.012 \\
\hline
\end{tabular}

The final multivariable model included variables that were significantly $(\mathrm{P}<0.05)$ associated with the cardiovascular risk factor in question. The following variables were tested age, gender, smoking, family history of coronary heart disease, previous cardiovascular disease, body mass index, transmission mode, CD4 cell count, HIV RNA, previous AIDS. PI, protease inhibitor; NNRTI, non-nucleoside reverse transcriptase inhibitor; NRTI, nucleoside reverse transcriptase inhibitor; OR, odds ratio; CI, confidence interval; HDL, high density lipoprotein; TG, triglycerides.

justed risk of elevated triglycerides decreased with increasing HIV-1 RNA [OR (95\% CI), $0.86(0.78$ to 0.95$)$ per $1 \log _{10}$ increase; $\mathrm{P}=0.002]$, and there was also a significant association with CD4 cell count [OR, 1.20 (IC95\%: 1.10 to 1.31) per $\log _{2}$ CD4 twofold increase; $\left.\mathrm{P}<0.001\right]$.

\section{Serum HDL-Cholesterol}

None of the current regimens were associated with an increased risk of low HDL-cholesterol (Tables 2-4) in the multivariate logistic model. The associations of CD4 cell count and HIV-1 viral load were similar for the absolute value of HDL-cholesterol and for total cholesterol.

\section{Hypertension}

More than $20 \%$ of the study population had hypertension. In a univariable logistic model, regimens containing NNRTI, PI or both drug classes were associated with a non statistically different risk of being hypertensive (Tables 3 and $\mathbf{4}$ ) which was explained by a strong correlation of hypertension with other factors (age, sex and BMI).

\section{Diabetes}

The overall prevalence of diabetes was $7.3 \%$. In a univariable model, all regimens were associated with a differen- 
Table 5. Cardiovascular Risk Factors in the VACH Cohort Population According to the Presence of Fat Redistribution

\begin{tabular}{|c|c|c|c|c|}
\hline & Without LD (n=1938) & With LD (n=420) & Total $(n=2358)$ & $\mathbf{p}$ \\
\hline Age (years), median [IQR] & $\begin{array}{c}39.00 \\
{[35.00,44.00]}\end{array}$ & $\begin{array}{c}41.00 \\
{[37.00,46.00]}\end{array}$ & $\begin{array}{c}40.00 \\
{[35.00,44.00]}\end{array}$ & $<0.0001$ \\
\hline Age $>45$ years male, $>55$ female, $\mathrm{n}(\%)[95 \% \mathrm{CI}]$ & $\begin{array}{c}331(17.1 \%) \\
{[15.4 \%, 18.8 \%]}\end{array}$ & $\begin{array}{c}95(22.6 \%) \\
{[18.7 \%, 26.9 \%]}\end{array}$ & $\begin{array}{c}426(18.1 \%) \\
{[16.5 \%, 19.7 \%]}\end{array}$ & 0.0076 \\
\hline Body mass index, median [IQR] & $\begin{array}{c}23.80 \\
{[21.72,25.65]}\end{array}$ & $\begin{array}{c}23.83 \\
{[21.84,25.85]}\end{array}$ & $\begin{array}{c}23.82 \\
{[21.75,25.67]}\end{array}$ & 0.5305 \\
\hline Body mass index $>30 \mathrm{~kg} / \mathrm{m} 2$ median, $\mathrm{n}(\%)[95 \% \mathrm{CI}]$ & $\begin{array}{c}74(3.8 \%) \\
{[3.0 \%, 4.8 \%]}\end{array}$ & $\begin{array}{c}8(1.9 \%) \\
{[0.8 \%, 3.7 \%]}\end{array}$ & $\begin{array}{c}82(3.5 \%) \\
{[2.8 \%, 4.3 \%]}\end{array}$ & 0.0523 \\
\hline Current smoker, $\mathrm{n}(\%)[95 \% \mathrm{CI}]$ & $\begin{array}{c}1141(58.9 \%) \\
{[56.6 \%, 61.1 \%]}\end{array}$ & $\begin{array}{c}256(61.0 \%) \\
{[56.1 \%, 65.6 \%]}\end{array}$ & $\begin{array}{c}1397(59.2 \%) \\
{[57.2 \%, 61.2 \%]}\end{array}$ & 0.4322 \\
\hline Sex (female), n (\%) [95\%CI] & $\begin{array}{c}439(22.7 \%) \\
{[20.8 \%, 24.6 \%]}\end{array}$ & $\begin{array}{c}83(19.8 \%) \\
{[16.1 \%, 23.9 \%]}\end{array}$ & $\begin{array}{c}522(22.1 \%) \\
{[20.5 \%, 23.9 \%]}\end{array}$ & 0.1959 \\
\hline Clinical AIDS, n (\%) [95\%CI] & $\begin{array}{c}630(32.5 \%) \\
{[30.4 \%, 34.6 \%]}\end{array}$ & $\begin{array}{c}158(37.6 \%) \\
{[33.0 \%, 42.4 \%]}\end{array}$ & $\begin{array}{c}788(33.4 \%) \\
{[31.5 \%, 35.4 \%]}\end{array}$ & 0.0441 \\
\hline Clinical or Immunologic AIDS, n (\%) [95\%CI] & $\begin{array}{c}1100(56.8 \%) \\
{[54.5 \%, 59.0 \%]}\end{array}$ & $\begin{array}{c}268(63.8 \%) \\
{[59.0 \%, 68.4 \%]}\end{array}$ & $\begin{array}{c}1368(58.0 \%) \\
{[56.0 \%, 60.0 \%]}\end{array}$ & 0.0080 \\
\hline CD4 cell count (x 106/1), median [IQR)] & $\begin{array}{c}414.00 \\
{[249.50,628.50]}\end{array}$ & $\begin{array}{c}480.00 \\
{[310.00,713.00]}\end{array}$ & $\begin{array}{c}426.00 \\
{[256.00,651.00]}\end{array}$ & $<0.0001$ \\
\hline Nadir CD4 cell count (x 106/1), median [IQR)] & $\begin{array}{c}141.00 \\
{[49.00,252.00]}\end{array}$ & $\begin{array}{c}130.00 \\
{[47.00,240.00]}\end{array}$ & $\begin{array}{c}140.00 \\
{[48.00,250.00]}\end{array}$ & 0.3251 \\
\hline Time from HIV diagnosis (years), median [IQR)] & $\begin{array}{c}7.21 \\
{[4.02,12.47]}\end{array}$ & $\begin{array}{c}9.08 \\
{[6.26,13.98]}\end{array}$ & $\begin{array}{c}7.46 \\
{[4.42,12.75]}\end{array}$ & $<0.0001$ \\
\hline HIV-1 RNA ( $\log 10)$, median [IQR)] & $\begin{array}{c}1.70 \\
{[1.69,2.24]}\end{array}$ & $\begin{array}{c}1.69 \\
{[1.69,1.85]}\end{array}$ & $\begin{array}{c}1.69 \\
{[1.69,2.17]}\end{array}$ & 0.0011 \\
\hline ART exposure (years), median [IQR)] & $\begin{array}{c}4.01 \\
{[1.72,5.95]}\end{array}$ & $\begin{array}{c}5.51 \\
{[3.75,6.85]}\end{array}$ & $\begin{array}{c}4.29 \\
{[1.98,6.19]}\end{array}$ & $<0.0001$ \\
\hline HAART exposure (years), median [IQR)] & $\begin{array}{c}3.80 \\
{[1.65,5.52]}\end{array}$ & $\begin{array}{c}5.29 \\
{[3.70,6.56]}\end{array}$ & $\begin{array}{c}4.10 \\
{[1.90,5.79]}\end{array}$ & $<0.0001$ \\
\hline Exposure to PI, n (\%) $[95 \% \mathrm{CI}]$ & $\begin{array}{c}1363(70.3 \%) \\
{[68.2 \%, 72.4 \%]}\end{array}$ & $\begin{array}{c}348(82.9 \%) \\
{[78.9 \%, 86.3 \%]}\end{array}$ & $\begin{array}{c}1711(72.6 \%) \\
{[70.7 \%, 74.4 \%]}\end{array}$ & $<0.0001$ \\
\hline Duration (years), median [IQR)] & $\begin{array}{c}2.42 \\
{[0.00,5.53]}\end{array}$ & $\begin{array}{c}5.25 \\
{[1.98,6.64]}\end{array}$ & $\begin{array}{c}3.26 \\
{[0.00,5.80]}\end{array}$ & $<0.0001$ \\
\hline Exposure to NNRTIs, $\mathrm{n}(\%)[95 \% \mathrm{CI}]$ & $\begin{array}{c}1283(66.2 \%) \\
{[64.0 \%, 68.3 \%]}\end{array}$ & $\begin{array}{c}297(70.7 \%) \\
{[66.1 \%, 75.0 \%]}\end{array}$ & $\begin{array}{c}1580(67.0 \%) \\
{[65.1 \%, 68.9 \%]}\end{array}$ & 0.0746 \\
\hline Duration (years), median [IQR)] & $\begin{array}{c}1.33 \\
{[0.00,3.45]}\end{array}$ & $\begin{array}{c}2.49 \\
{[0.00,4.17]}\end{array}$ & $\begin{array}{c}1.51 \\
{[0.00,3.64]}\end{array}$ & $<0.0001$ \\
\hline Exposure to NRTIs, n (\%) $[95 \% \mathrm{CI}]$ & $\begin{array}{c}1935(99.8 \%) \\
{[99.5 \%, 100.0 \%]}\end{array}$ & $\begin{array}{c}420(100.0 \%) \\
{[99.3 \%, 100.0 \%]}\end{array}$ & $\begin{array}{c}2355(99.9 \%) \\
{[99.6 \%, 100.0 \%]}\end{array}$ & 0.4198 \\
\hline Duration (years), median [IQR)] & $\begin{array}{c}4.00 \\
{[1.72,5.94]}\end{array}$ & $\begin{array}{c}5.51 \\
{[3.73,6.85]}\end{array}$ & $\begin{array}{c}4.28 \\
{[1.98,6.19]}\end{array}$ & $<0.0001$ \\
\hline Family story of CVD, n (\%) [95\%CI] & $\begin{array}{c}236(12.2 \%) \\
{[10.8 \%, 13.7 \%]}\end{array}$ & $\begin{array}{c}70(16.7 \%) \\
{[13.2 \%, 20.6 \%]}\end{array}$ & $\begin{array}{c}306(13.0 \%) \\
{[11.6 \%, 14.4 \%]}\end{array}$ & 0.0131 \\
\hline Previous CVD, n (\%) [95\%CI] & $\begin{array}{c}33(1.7 \%) \\
{[1.2 \%, 2.4 \%]}\end{array}$ & $\begin{array}{c}7(1.7 \%) \\
{[0.7 \%, 3.4 \%]}\end{array}$ & $\begin{array}{c}40(1.7 \%) \\
{[1.2 \%, 2.3 \%]}\end{array}$ & 0.9586 \\
\hline Hypertension, n (\%) [95\%CI] & $\begin{array}{l}412(21.3 \%)[ \\
19.5 \%, 23.2 \%]\end{array}$ & $\begin{array}{c}127(30.2 \%) \\
{[25.9 \%, 34.9 \%]}\end{array}$ & $\begin{array}{c}539(22.9 \%) \\
{[21.2 \%, 24.6 \%]}\end{array}$ & $<0.0001$ \\
\hline Diabetes mellitus [\% (95\% CI) & $\begin{array}{c}121(6.2 \%) \\
{[5.2 \%, 7.4 \%]}\end{array}$ & $\begin{array}{c}51(12.1 \%) \\
{[9.2 \%, 15.7 \%]}\end{array}$ & $\begin{array}{c}172(7.3 \%) \\
{[6.3 \%, 8.4 \%]}\end{array}$ & $<0.0001$ \\
\hline Total cholesterol (mmol/l), median [IQR)] & $\begin{array}{c}4.87 \\
{[4.14,5.67]} \\
\end{array}$ & $\begin{array}{c}4.84 \\
{[3.96,5.67]} \\
\end{array}$ & $\begin{array}{c}4.87 \\
{[4.12,5.67]} \\
\end{array}$ & 0.2802 \\
\hline Total cholesterol > $6.2 \mathrm{mmol} / \mathrm{l}, \mathrm{n}(\%)[95 \% \mathrm{CI}]$ & $\begin{array}{c}280(14.4 \%) \\
{[12.9 \%, 16.1 \%]}\end{array}$ & $\begin{array}{c}66(15.7 \%) \\
{[12.4 \%, 19.6 \%]}\end{array}$ & $\begin{array}{c}346(14.7 \%) \\
{[13.3 \%, 16.2 \%]}\end{array}$ & 0.5061 \\
\hline
\end{tabular}




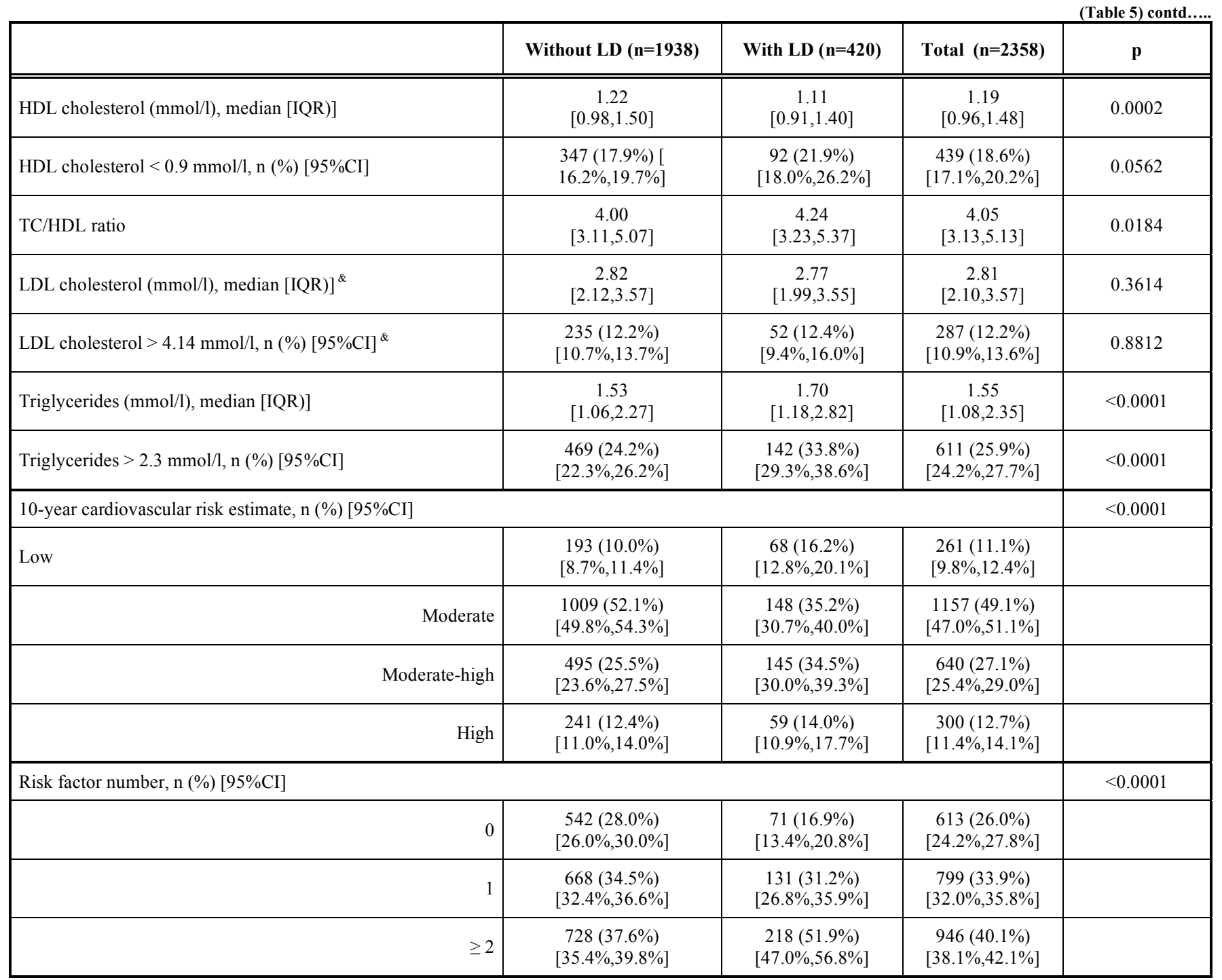

Descriptive statistics are $\mathrm{n}(\%)[95 \% \mathrm{CI}]$ for qualitative, and median [IQR] for qualitative variables. $\mathrm{LD}=$ lipodystrophy, AIDS $=$ acquired immunodeficiency syndrome, ART $=$ antiretroviral therapy, HAART = highly active antiretroviral therapy, $\mathrm{CVD}=$ cardiovascular disease, $\mathrm{PI}=$ protease inhibitor; $\mathrm{NNRTI}=$ non-nucleoside reverse transcriptase inhibitor $\mathrm{NRTI}=$ nucleoside reverse transcriptase inhibitor; $\mathrm{OR}=$ odds ratio; $\mathrm{CI}=$ confidence interval; $\mathrm{HDL}=$ high density lipoprotein; $\mathrm{TC}=$ total cholesterol.

\& Percentage of missingness $<1 \%$; null percentage for the rest of variables.

tial risk of diabetes (Tables 3 and 4). The adjusted multivariate increment of risk per year of exposure was $8 \%$ for NRTI $(\mathrm{p}=0.011), 10 \%$ for NNRTI $(\mathrm{p}=0.031)$ and $4 \%$ for PI $(0.243)$ (Table 4).

\section{Body Composition}

In all regimen groups there were few obese patients (Table 2). Antiretroviral therapy was highly associated with the presence of clinical lipodystrophy, with the highest risk among patients receiving a regimen containing all three drug classes (Tables 3 and 4). When assessed as an explanatory variable, lipodystrophy was associated with the presence of several of the CVD risk factors discussed above (Table 5). In a multivariable model including the total study population, and adjusting for co-variables as listed in Table 5, the adjusted OR for the association of lipodystrophy with elevated total cholesterol was $0.90(0.65-1.23 ; \mathrm{P}=0.494)$, elevated triglycerides $1.36(1.06-1.75 ; \mathrm{P}=0.017)$ and decreased HDL
1.19 (0.90.1.58; $\mathrm{P}=0.214)$. The presence of lipodystrophy was associated with an increased risk of hypertension and diabetes OR, $1.47(95 \% \mathrm{CI}, 1.14-1.90 ; \mathrm{P}=0.003)$ and 1.71 (95\%CI, 1.17-2.49, $\mathrm{P}=0.005)$, respectively.

\section{Cardiovascular Risk Assessment}

Ten-year CHD risk estimates for HIV-1-infected subjects treated with HAART in the VACH cohort are shown in Table 6. There was no clustering of CHD risk for any treatment group. The 10-year CHD of moderate-high or high risk estimate was significantly increased among HIV-1-infected patients with fat redistribution, compared with that for those with no redistribution: $48.5 \%$ versus $37.9 \%,(P<0.001)$ (Table 5). Ten-year CHD risk estimates were studied by current HAART regimen (Table 6) and by cumulated ART exposure (Table 4). Predictors of a moderate-high CVD risk estimate obtained from the multivariate logistic model were: OR (95\%CI; P value): $0.63(0.28-1.42 ; \mathrm{P}=0.266), 1.13$ 
Table 6. Cardiovascular Risk Assessment and Need for Lipid-Lowering Therapy (LLT) in the VACH Cohort Population According to Current Usage of Antiretroviral Therapy (ART) Drug Classes

\begin{tabular}{|c|c|c|c|c|c|c|}
\hline & $\begin{array}{l}\text { NRTI Only } \\
(\mathrm{n}=\mathbf{2 3 3})\end{array}$ & $\begin{array}{l}\text { Combination with } \\
\text { NNRTI }(\mathrm{n}=1094)\end{array}$ & $\begin{array}{l}\text { Combination with } \\
\text { PI }(n=990)\end{array}$ & $\begin{array}{c}\text { Combination with } \\
\text { PI plus NNRTI }(n=41)\end{array}$ & $\begin{array}{c}\text { Total } \\
(\mathrm{n}=\mathbf{2 3 5 8})\end{array}$ & $\mathbf{p}$ \\
\hline \multicolumn{6}{|c|}{ Risk factor number, n (\%) [95\%CI] } & 0.0001 \\
\hline 0 & $\begin{array}{c}42(18.0 \%) \\
{[13.3 \%, 23.6 \%]}\end{array}$ & $\begin{array}{c}326(29.8 \%) \\
{[27.1 \%, 32.6 \%]}\end{array}$ & $\begin{array}{c}228(23.0 \%) \\
{[20.4 \%, 25.8 \%]}\end{array}$ & $\begin{array}{c}17(41.5 \%) \\
{[26.3 \%, 57.9 \%]}\end{array}$ & $\begin{array}{c}613(26.0 \%) \\
{[24.2 \%, 27.8 \%]}\end{array}$ & \\
\hline 1 & $\begin{array}{c}78(33.5 \%) \\
{[27.4 \%, 39.9 \%]}\end{array}$ & $\begin{array}{c}363(33.2 \%) \\
{[30.4 \%, 36.1 \%]}\end{array}$ & $\begin{array}{c}345(34.8 \%) \\
{[31.9 \%, 37.9 \%]}\end{array}$ & $\begin{array}{c}13(31.7 \%) \\
{[18.1 \%, 48.1 \%]}\end{array}$ & $\begin{array}{c}799(33.9 \%) \\
{[32.0 \%, 35.8 \%]}\end{array}$ & \\
\hline$\geq 2$ & $\begin{array}{c}113(48.5 \%) \\
{[41.9 \%, 55.1 \%]}\end{array}$ & $\begin{array}{c}405(37.0 \%) \\
{[34.2 \%, 40.0 \%]}\end{array}$ & $\begin{array}{c}417(42.1 \%) \\
{[39.0 \%, 45.3 \%]}\end{array}$ & $\begin{array}{c}11(26.8 \%) \\
{[14.2 \%, 42.9 \%]}\end{array}$ & $\begin{array}{c}946(40.1 \%) \\
{[38.1 \%, 42.1 \%]}\end{array}$ & \\
\hline \multicolumn{7}{|c|}{ 10-yr Cardiovascular risk estimate, $\mathrm{n}(\%)[95 \% \mathrm{CI}]$} \\
\hline Low & $\begin{array}{c}107(45.9 \%) \\
{[39.4 \%, 52.6 \%]}\end{array}$ & $\begin{array}{c}548(50.1 \%) \\
{[47.1 \%, 53.1 \%]}\end{array}$ & $\begin{array}{c}475(48.0 \%) \\
{[44.8 \%, 51.1 \%]}\end{array}$ & $\begin{array}{c}27(65.9 \%) \\
{[49.4 \%, 79.9 \%]}\end{array}$ & $\begin{array}{c}1157(49.1 \%) \\
{[47.0 \%, 51.1 \%]}\end{array}$ & 0.0184 \\
\hline Moderate & $\begin{array}{c}65(27.9 \%) \\
{[22.2 \%, 34.1 \%]}\end{array}$ & $\begin{array}{c}280(25.6 \%) \\
{[23.0 \%, 28.3 \%]}\end{array}$ & $\begin{array}{c}289(29.2 \%) \\
{[26.4 \%, 32.1 \%]}\end{array}$ & $\begin{array}{c}6(14.6 \%) \\
{[5.6 \%, 29.2 \%]}\end{array}$ & $\begin{array}{c}640(27.1 \%) \\
{[25.4 \%, 29.0 \%]}\end{array}$ & \\
\hline Moderate-high & $\begin{array}{c}34(14.6 \%) \\
{[10.3 \%, 19.8 \%]}\end{array}$ & $\begin{array}{c}124(11.3 \%) \\
{[9.5 \%, 13.4 \%]}\end{array}$ & $\begin{array}{c}138(13.9 \%) \\
{[11.8 \%, 16.3 \%]}\end{array}$ & $\begin{array}{c}4(9.8 \%) \\
{[2.7 \%, 23.1 \%]}\end{array}$ & $\begin{array}{c}300(12.7 \%) \\
{[11.4 \%, 14.1 \%]}\end{array}$ & \\
\hline High & $\begin{array}{c}27(11.6 \%) \\
{[7.8 \%, 16.4 \%]}\end{array}$ & $\begin{array}{c}142(13.0 \%) \\
{[11.0 \%, 15.1 \%]}\end{array}$ & $\begin{array}{c}88(8.9 \%) \\
{[7.2 \%, 10.8 \%]}\end{array}$ & $\begin{array}{c}4(9.8 \%) \\
{[2.7 \%, 23.1 \%]}\end{array}$ & $\begin{array}{c}261(11.1 \%) \\
{[9.8 \%, 12.4 \%]}\end{array}$ & \\
\hline \multicolumn{7}{|c|}{ Lipid-lowering therapy, n (\%) $[95 \% \mathrm{CI}]^{\text {\& }}$} \\
\hline Needed & $\begin{array}{c}26(11.3 \%) \\
{[7.5 \%, 16.1 \%]}\end{array}$ & $\begin{array}{c}201(18.5 \%) \\
{[16.2 \%, 20.9 \%]}\end{array}$ & $\begin{array}{c}177(17.9 \%) \\
{[15.6 \%, 20.5 \%]}\end{array}$ & $\begin{array}{c}11(26.8 \%) \\
{[14.2 \%, 42.9 \%]}\end{array}$ & $\begin{array}{c}415(17.7 \%) \\
{[16.2 \%, 19.3 \%]}\end{array}$ & 0.0243 \\
\hline Current use & $\begin{array}{c}6(2.6 \%) \\
{[1.0 \%, 5.5 \%]}\end{array}$ & $\begin{array}{c}36(3.3 \%) \\
{[2.3 \%, 4.5 \%]}\end{array}$ & $\begin{array}{c}21(2.1 \%) \\
{[1.3 \%, 3.2 \%]}\end{array}$ & $\begin{array}{c}0(0.0 \%) \\
{[0.0 \%, 7.0 \%]}\end{array}$ & $\begin{array}{c}63(2.7 \%) \\
{[2.1 \%, 3.4 \%]}\end{array}$ & 0.2725 \\
\hline \multicolumn{6}{|l|}{ Type of therapy, n (\%) $[95 \% \mathrm{CI}]$} & 0.3161 \\
\hline None & $\begin{array}{c}227(97.4 \%) \\
{[94.5 \%, 99.0 \%]}\end{array}$ & $\begin{array}{c}1058(96.7 \%) \\
{[95.5 \%, 97.7 \%]}\end{array}$ & $\begin{array}{c}969(97.9 \%) \\
{[96.8 \%, 98.7 \%]}\end{array}$ & $\begin{array}{c}41(100.0 \%) \\
{[93.0 \%, 100.0 \%]}\end{array}$ & $\begin{array}{c}2295(97.3 \%) \\
{[96.6 \%, 97.9 \%]}\end{array}$ & \\
\hline Fibrates & $\begin{array}{c}3(1.3 \%) \\
{[0.3 \%, 3.7 \%]}\end{array}$ & $\begin{array}{c}12(1.1 \%) \\
{[0.6 \%, 1.9 \%]}\end{array}$ & $\begin{array}{c}12(1.2 \%) \\
{[0.6 \%, 2.1 \%]}\end{array}$ & $\begin{array}{c}0(0.0 \%) \\
{[0.0 \%, 7.0 \%]}\end{array}$ & $\begin{array}{c}27(1.1 \%) \\
{[0.8 \%, 1.7 \%]}\end{array}$ & \\
\hline Statines & $\begin{array}{c}3(1.3 \%) \\
{[0.3 \%, 3.7 \%]}\end{array}$ & $\begin{array}{c}24(2.2 \%) \\
{[1.4 \%, 3.2 \%]}\end{array}$ & $\begin{array}{c}9(0.9 \%) \\
{[0.4 \%, 1.7 \%]}\end{array}$ & $\begin{array}{c}0(0.0 \%) \\
{[0.0 \%, 7.0 \%]}\end{array}$ & $\begin{array}{c}36(1.5 \%) \\
{[1.1 \%, 2.1 \%]}\end{array}$ & \\
\hline
\end{tabular}

Descriptive statistics are $\mathrm{n}(\%)[95 \% \mathrm{CI}]$ for qualitative, and median [IQR] for qualitative variables.

${ }^{\&}$ Percentage of missingness $<1 \%$; null percentage for the rest of variables.

(0.52-2.46; $\mathrm{P}=0.756)$, and $1.91(0.48-7.57 ; \mathrm{P}=0.359)$ for NRTI, PI, NNRTI+PI current ART regimens, respectively.

\section{Lipid-Lowering Therapy (LLT)}

The proportion of patients who fulfilled criteria for being treated with LLT were $11.3 \%, 18.5 \%, 17.9 \%$, and $26.8 \%$, respectively for patients treated with NRTI only or with NNRTI, PI or NNRTI and PI, respectively ( $\mathrm{P}=0.0243)$. However, only between a fourth and a fifth of those patients were currently receiving LLT.

\section{DISCUSSION}

In the VACH population we have observed a high prevalence of multiple risk factors for CVD. VACH has the strength of having included more than 2000 treated patients with details concerning CVD risk factors, with an almost insignificant proportion of missing data. Similar to other studies [13], we found that regimens containing drugs from both the PI and NNRTI classes were associated with the highest prevalence of dyslipidemia. Furthermore, we also observed that hypercholesterolemia was associated with a higher CD4 cell count, a lower HIV-1 plasma viral load, the presence of clinical signs of lipodystrophy and older age. These findings, and those of others [13, 20], suggest that immune reconstitution phenomena may play some role in the development of antiretroviral-associated dyslipidemia.

However, our findings have inherent limitations. Firstly, our findings are not applicable to other populations since the diversity of the study population, including women, minorities and means of acquiring HIV-1 infection, implies that the study may not be representative of the HIV-1-infected population in other industrialized countries. Furthermore, the Mediterranean basin is an area of low incidence in terms of coronary heart disease and its risk factors, which may make comparisons with similar studies difficult [21, 22]. However, our study suggests that even in such an environment, HIV-1infected patients on HAART develop comparable metabolic disturbances to other geographically-based cohorts [23-25]. Since information concerning certain other potential risk factors for CVD, such as diet, physical activity and genetic 
(a)

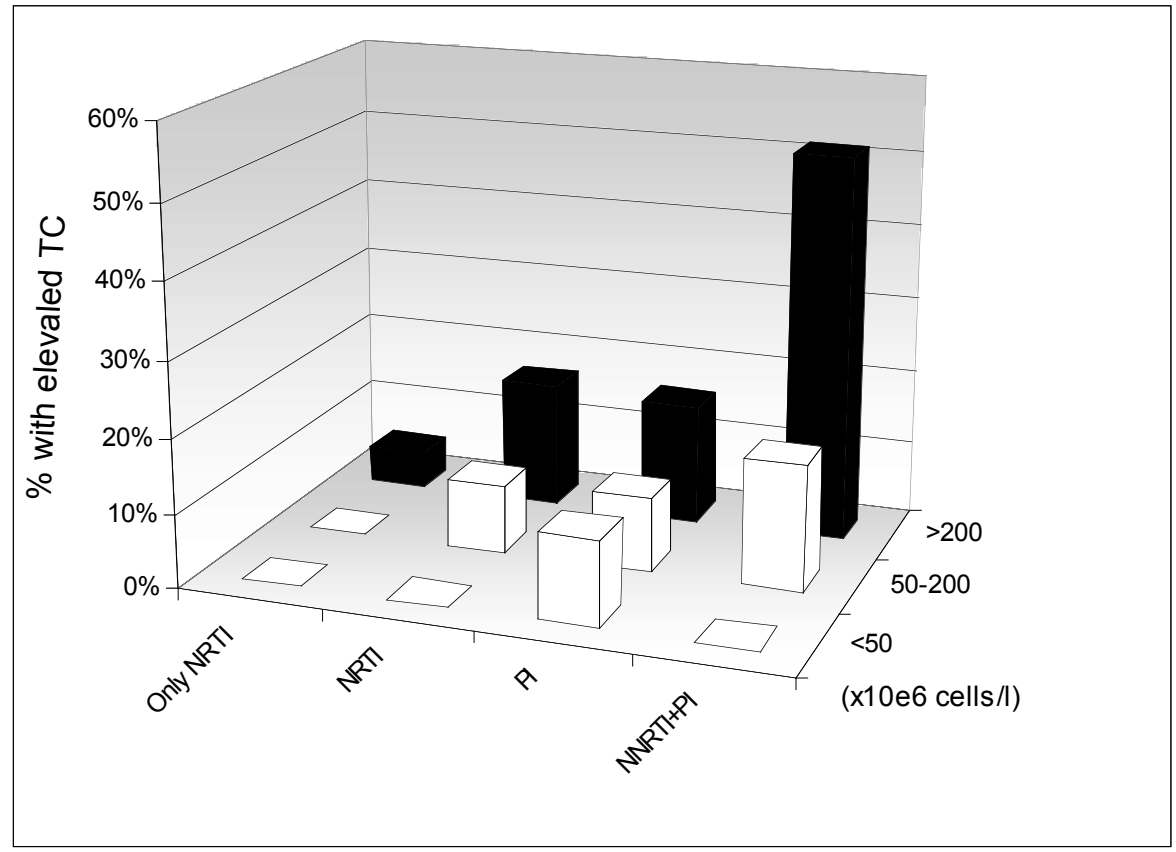

(b)

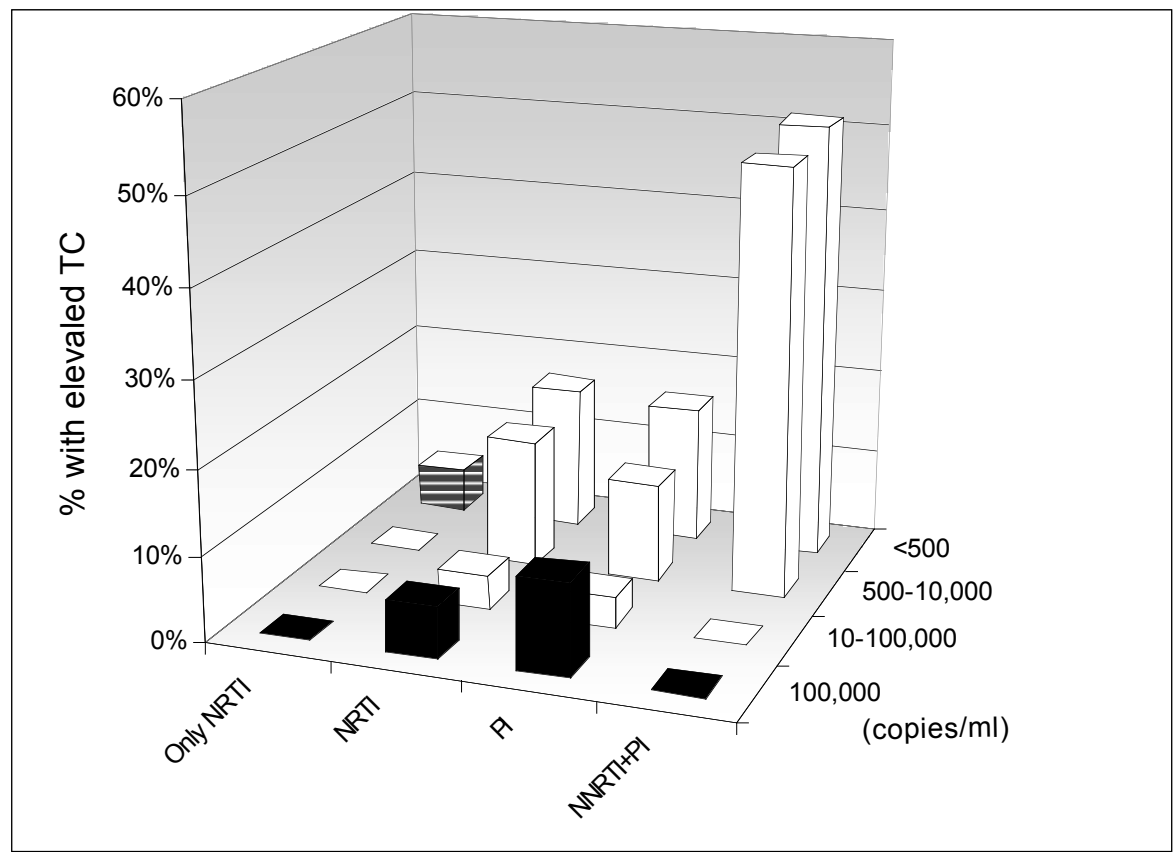

Fig. (1). Prevalence of elevated total cholesterol ( $>6.2 \mathrm{mmol} / \mathrm{l})$ according to current antiretroviral therapy (ART), CD4 cell count (a) and HIV RNA (b) at baseline. The four ART categories are: NRTI (currently receiving only NRTI), NNRTI (currently receiving NNRTI and NRTI but not PI), PI (currently receiving PI and NRTI but not NNRTI), and PI/NNRTI (currently receiving PI, NNRTI and NRTI).

factors, was not collected in our study, this may restrict the validity of our findings only to populations similar to ours.

Other limitations are related to the observational design of the study. Firstly, the results presented are only associations from which no conclusions regarding causality can or should be drawn. Secondly, due to the observational design of the study, many measurements (blood pressure, lipid levels) are expected not to be always conducted in a uniform manner. However, international and national standardization of serum lipid measurements have been accomplished throughout the guidelines of the NCEP ATP II and the Spanish Society of Hypertension [19, 26]. On the other hand, the relatively low proportion of missing data should be noted (Table 1), which implies that the prevalence of the individual risk factors is precise. 
Dyslipidemia was most strongly correlated with antiretroviral regimens currently being used, and less with a history of previous exposure to the different drug classes. This is consistent with previous reports, in which the PI-associated dyslipidemia occurred shortly after beginning therapy [27, 28] and it is also consistent with studies showing that a switch from PI to NNRTI-based or NRTI-only regimens is associated with attenuation or resolution of dyslipidemia [29, $30]$. The average increases in lipid levels [2, 13, 31, 32], comparing levels during PI therapy with either pre-therapy levels or levels in PI-naïve HIV-1-infected patients, were $28 \%$ for total cholesterol and $96 \%$ for triglycerides. We observed no difference in risk of low HDL-cholesterol among patients treated with PI, NRTI or NNRTIs, data reported by others as well $[13,26]$. Duration of PI therapy did not influence the level of HDL cholesterol [32], whereas duration of NRTI was associated with a higher risk of low HDLcholesterol.

The association between NNRTI-containing regimens and dyslipidemia has rarely been reported [13], although, in phase I studies of efavirenz in HIV-1-uninfected subjects revealed increases in total cholesterol levels of $10-20 \%$ in some subjects [3], whereas cohort and trial data suggest that $18-20 \%$ of NNRTI-treated patients develop dyslipidemia [23-25, 34]. Although a different lipid profile between the two NNRTIs used has been frequently claimed [30], no differences were reported in HIV-1-infected individuals [35]. In concordance with our results, an increase in HDL-cholesterol with NNRTI has been reported [36]. Consistent with previous reports, NRTI-only therapy was associated with lower rates of elevated total cholesterol [35, 37].

We found a strong association between elevated total cholesterol level and higher CD4 cell counts, which was present within each treatment category. Nevertheless, within each CD4 cell count stratum, the effect of antiretroviral therapy was clearly observed, which indicates that the effect of antiretroviral substances certainly cannot solely be explained by a reversal to 'normal' pre-disease cholesterol levels as a result of improved cellular immunity. The level of HDLcholesterol, although to a lesser extent, likewise increased with more conserved cellular immunity, consistent with observations in the pre-HAART era [38]. For total cholesterol, the association with HIV-1 viral load was the inverse of the association with CD4 cell count. The latter has also been reported from other studies [39]. However, it could also be possible that these findings merely reflect the cumulative exposure to antiretrovirals.

In the VACH study, we have observed a high prevalence of other known and potential CVD risk factors among patients receiving either PI or NNRTI, including cigarette smoking, diabetes, hypertension and altered body composition. The overall prevalence of diabetes mellitus in the VACH study was $7.3 \%$, similar to other studies that have shown an association between diabetes mellitus and use of PI [1, 27, 31], and recently with NRTI exposure [40]. A few studies have reported an increased prevalence of hypertension in PI-treated patients [3, 4] or in conjunction with lipodystrophy [41]. In our study, the associations between antiretroviral drug regimens and hypertension in univariable logistic models were no longer present after adjustment for other factors associated with hypertension. This is in odds with data from the DAD cohort showing no deleterious effect of any class of antiretrovirals on blood pressure [42]. There was a marked association between dyslipidemia and several of the other CVD risk factors on the one hand and clinical lipodystrophy on the other [43]. Current guidelines for the management of dyslipidemia in HIV-1-infected patients have been developed [44]. Assuming that dyslipidemia in HIV-1-infected will have similar long-term consequences to dyslipidemia in the general population, these guidelines also assume that the benefits of lipid lowering interventions will also extend to HIV-1-infected persons, and thus these are based on CVR stratification while the goals to be achieved are based on target values for LDL cholesterol and non-HDL cholesterol [43]. Despite that, we found that only between a fourth and a fifth of patients in our cohort needing LLT were effectively being treated, a figure somwwhat higher than those reported in previous years [34]. This finding may have many causes, but among them the lack of LLT knowledge by HIV-1 treating physicians might be considered. In a recent report, it was shown that when dyslipidemic HIV-1-infected patients were addressed to a specialized lipid clinic, the percentage of those being treated with LLT significantly increased [45].

Recently, the issue of how young persons, with a low absolute risk may be at a substantially relative higher has been addressed in the European guidelines [46], and this is particularly the case for HIV-1-infected patients because even if its absolute risk is low, it may still be 10-12 times higher than that of a person with low risk factor, usually because the addition of cumulative risk factors. The present study shows that the use of potent antiretroviral therapy resulting in more profound virus suppression and more preserved immunity, was associated with a high both relative and absolute risk of exhibiting risk factors for CHD. How these projections will translate to clinical events of CHD is difficult to predict, since it is assumed that there presumably will be a time lag from when factors known to accelerate the atherosclerotic process are induced and until clinical manifestations of atherosclerotic vascular disease occur. Present evidences suggest that HIV-1-infected patients have a $27 \%$ yearly risk of developing a cardiovascular event [47]. As many of these factors are likely to act synergistically, together with the underlying HIV-1 infection itself the time-lag cannot be reliably assessed. However, ongoing studies such as DAD [13] may provide fruitful comparisons between expected and observed CVD event rates [48]. Clearly, the management of CVR factors among our HIV-1-infected patients needs to be improved, and must follow two paths: a continued need for developing less harmful and better tolerated effective treatments for HIV-1 infection together with a better knowledge of pharmaceutical and non-pharmaceutical measures directed at reducing CVD risk by HIV-1 physicians. Given the high prevalence of smoking among our patients, strategies to quit smoking will likely prove costeffective in preventing CVD in HIV-1-infected patients. 


\section{JUSTIFICATION OF AUTHORSHIP}

Pere Domingo, Ignacio Suárez-Lozano, and Myriam Garrido were in charge of the design, coordination and wrote the article. Pompeyo Viciana, Fernando Lozano, Alberto Terron, MJosé Galindo, Juan Gonzalez, Pere Domingo, Esteban Ribera, Antonio Vergara, Ramón Teira, Paloma Geijo, Jaime Cosín, Bernardino Roca, Belen de la Fuente, Trinitario Sanchez, contributed to de design of the computer application, data entry for the study, local coordination and bring up ideas to complete the final version of the manuscript. Juan R Lacalle and Ferran Torres made the statistic analyses.

\section{FINANCIAL SUPPORT}

This work was possible through an unrestricted grant from Bristol, Myers \& Squibb.

\section{APPENDIX: CUTT-OFF VALUES FOR SPECIFICA- TION OF CVD RISK FACTORS IN HIV-1-INFECTED PATIENTS}

The specification of risk factors is as follows. (i) Dyslipidemia: defined as elevated total cholesterol $>6.2 \mathrm{mmol} / \mathrm{l}$ (240 mg/dl), and/or decreased HDL-cholesterol $<0.9 \mathrm{mmol} / 1$ $(35 \mathrm{mg} / \mathrm{dl})$, and/or elevated triglycerides $>2.3 \mathrm{mmol} / \mathrm{l}(200$ $\mathrm{mg} / \mathrm{dl}$ ). [The cut-offs applied are based on cut-offs for high risk for CVD in the NCEP guidelines.] (ii) Older age: age $>$ 45 years for men and $>55$ for women. (iii) Family history of coronary heart disease: first-degree relative with myocardial infarction before age 50. (iv) Previous CVD: patients' own previous experience of myocardial infarction and/or stroke. (v) Hypertension: elevated systolic blood pressure $>140$ $\mathrm{mmHg}$ and/or elevated diastolic blood pressure $>90 \mathrm{mmHg}$, or usage of anti-hypertensive drugs. (vi) Diabetes: history of diabetes or usage of anti-diabetic therapy. (vii) Body mass index (BMI) was stratified in four categories: underweight $\left(\mathrm{BMI}<18 \mathrm{~kg} / \mathrm{m}^{2}\right.$ ), normal weight (BMI, $18-26 \mathrm{~kg} / \mathrm{m}^{2}$ ), overweight (BMI, 26.1-30 kg/m ${ }^{2}$ ) and obesity (BMI,> 30 $\mathrm{kg} / \mathrm{m}^{2}$ ). Obesity was considered a cardiovascular risk factor. (viii) Smoking: current cigarette smoking at inclusion in the study. (ix) Presence of clinical lipodystrophy was defined as either characteristic fat loss (from the face and/or extremities), central fat gain (abdominal and/or cervicodorsal) or mixed (at least one sign each of fat loss and central fat gain), as judged by the treating physician.

The specification of therapies to manage CVD risk factors were as follows: a) patients taking lipid-lowering agents b) anti-hypertensive medication c) anti-diabetic agents or insulin (derivatives) d) platelet aggregation inhibitors, and e) smoking cessation therapy.

\section{REFERENCES}

[1] Behrens G, Dejam A, Schmidt H, Balks HJ, Brabant G, Korner T, et al. (1999). Impaired glucose tolerance, beta cell function and lipid metabolism in HIV patients under treatment with protease inhibitors. AIDS. 13: F63-F70.

[2] Carr A, Samaras K, Burton S, Law M, Freund J, Chisholm DJ, et al. (1998). A syndrome of peripheral lipodystrophy, hyperlipidaemia and insulin resistance in patients receiving HIV protease inhibitors. AIDS. 12: F51-F58.

[3] Crane HM, Van Rompaey SE, Kitahata MM. (2006). Antiretroviral medications associated with elevated blood pressure among pa- tients receiving highly active antiretroviral therapy. AIDS. 20: 1019-1026.

[4] Cattelan AM, Trevenzoli M, Sasset L, Rinaldi L, Balasso V, Cadrobbi P. (2001). Indinavir and systemic hypertension. AIDS. 15: 805-807.

[5] Depairon M, Chessex S, Sudre P, Rodondi N, Doser N, Chave JP, et al. (2001). Swiss HIV Cohort Study. Premature atherosclerosis in HIV-infected individuals. Focus on protease inhibitor therapy. AIDS. 15: 329-334.

[6] Maggi P, Serio G, Epifani G, Fiorentino G, Saracino A, Fico C, et al. (2000). Premature lesions of the carotid vessels in HIV-1infected patients treated with protease inhibitors. AIDS. 14: F123128.

[7] Seminari E, Pan A, Voltini G, Carnevale G, Maserati R, Minoli L, et al. (2002). Assessment of atherosclerosis using carotid ultrasonography in a cohort of HIV-positive patients treated with protease inhibitors. Atherosclerosis. 162: 433-438.

[8] Stein JH, Klein MA, Bellehumeur JL, McBride PE, Wiebe DA, Otvos JD, et al. (2001). Use of human immunodeficiency virus-1 protease inhibitors is associated with atherogenic lipoprotein changes and endothelial dysfunction. Circulation. 104: 57-62.

[9] Mary-Krause M, Cotte L, Simon A, Partisani M, Costagliola D. (2003). Clinical Epidemiology Group from the French Hospital Database. Increased risk of myocardial infarction with duration of protease inhibitor therapy in HIV-infected men. AIDS. 17: 2479-86..

[10] Holmberg S, Moorman A, Williamson JM, Tong TC, Ward DJ, Wood KC, et al. (2002). HIV Outpatient Study (HOPS) investigators. Protease Inhibitor and cardiovascular outcomes in patients with HIV-1. Lancet. 360: 1747-1748.

[11] Sudano I, Spieker LE, Noll G, Corti R, Weber R, Luscher TF. (2006). Cardiovascular disease in HIV infection. Am Heart J. 15: 1147-55.

[12] El Sadr W, Reiss P, De Wit S, De Wit S, D'Arminio Monforte A, Thiébaut R, et al. Relationship between prolonged exposure to combination ART and myocardial infarction: effect of sex, age, and lipid changes: 2005: Boston, USA; 2005. p. 347.

[13] Friis-Moller N, Weber R, Reiss P, Thiebaut R, Kirk O, d'Arminio Monforte A, DAD study group. Cardiovascular disease risk factors in HIV patients: association with antiretroviral therapy: results from the D:A:D Study. AIDS. 17: 1179-1193.

[14] Suarez-Lozano I, Fajardo JM, Garrido M, Roca B, Garcia-Alcalde ML, Geijo P, et al. (2002). Epidemiological trends of HIV infection in Spain: preventative plans have to be oriented to new target populations (Spanish VACH Cohort). AIDS. 16: 2496-9.

[15] 1993 Revised Classification System for HIV Infection and Expanded Surveillance Case Definition for AIDS Among Adolescents and Adults. MMWR. (1992). 41(RR-17): 1-19.

[16] Wilson PW, D’Agostino, RB, Levy D, Belanger AM, Silbershatz H, Kannel WB. (1998). Prediction of coronary heart disease using risk factor categories. Circulation. 97: 1837-1847.

[17] Ramsay LE, Williams B, Johnston GD, MacGregor GA, Poston L, Potter JF, et al. (1999). British Hypertension Society Guidelines for Hypertension Management 1999: Summary. BMJ. 319: 630-635.

[18] Obesity: Preventing and Managing the Global Epidemic. (2000) Report of a WHO Consultation. World Health Organ Tech Rep Ser. 894: I-253.

[19] Summary of the Second Report of the National Cholesterol Education Program (NCEP) Expert Panel on Detection, Evaluation, and Treatment of High Blood Cholesterol in Adults (Adult Treatment Panel II). (1993). JAMA. 269: 3015-3023.

[20] Floris-Moore M, Howard AA, Lo Y, Arnsten JH, Santero N, Schoenbaum EE. Increased serum lipids are associated with higher CD4 lymphocyte count in HIV-infected women. HIV Med. 2006; 7: 421-430.

[21] Serra-Majem L, Ribas L, Tresserras R, Ngo J, Salleras L. (1995). How could changes in diet explain changes in coronary heart disease mortality in Spain? The Spanish paradox. Am J Clin Nutr. 61(6 Suppl): 1351S-1359S.

[22] Estruch R, Martinez-Gonzalez MA, Corella D, Salas-Salvado J, Ruiz-Gutierrez V, Covas MI, et al. (2006). Effects of a Mediterranean-style diet on cardiovascular risk factors: a randomized trial. Ann Intern Med. 145: 1-11.

[23] Fontas E, van Leth F, Sabin CA, Friis-Møller N, Rickenbach M, d'Arminio Monforte A, et al. (2004). Lipid profiles in HIV-infected patients receiving combination antiretroviral therapy: are different 
antiretroviral drugs associated with different lipid profiles. J Infect Dis. 189: 1056-1074.

[24] Fellay J, Boubaker K, Ledergerber B, Bernasconi E, Furrer H, Battegay M, et al. (2001). Prevalence of adverse events associated with potent antiretroviral treatment: Swiss HIV Cohort Study. Lancet. 358: 1322-1327.

[25] Bernasconi E, Boubaker K, Junghans C, Flepp M, Furrer HJ, Haensel A, et al. (2002). Abnormalities of body fat distribution in HIV-infected persons treated with antiretroviral drugs: The Swiss HIV Cohort Study. J Acquir Immune Defic Syndr. 31: 50-55.

[26] Sociedad Española de Hipertensión-Liga Española para la Lucha contra la Hipertensión Arterial SEH-LELHA. (2005). Guía española de hipertensión arterial 2005. Hipertensión. 22 (Suppl 2): 1-84

[27] Carr A, Samaras K, Thorisdottir A, Kaufmann GR, Chisholm DJ, Cooper DA. (1999). Diagnosis, prediction, and natural course of HIV-1 protease-inhibitor-associated lipodystrophy, hyperlipidaemia, and diabetes mellitus: a cohort study. Lancet. 353: 2093-2099.

[28] Purnell JQ, Zambon A, Knopp RH, Pizzuti DJ, Achari R, Leonard $\mathrm{JM}$, et al. (2000). Effect of ritonavir on lipids and post-heparin lipase activities in normal subjects. AIDS. 14: 51-57.

[29] Ruiz L, Negredo E, Domingo P, Paredes R, Francia E, Balague M, et al. (2001). Antiretroviral treatment simplification with nevirapine in protease inhibitor-experienced patients with hiv-associated lipodystrophy: 1-year prospective follow-up of a multicenter, randomized, controlled study. J Acquir Immune Defic Syndr. 27: 229236

[30] Martinez E, Arnaiz JA, Podzamczer D, Dalmau D, Ribera E, Domingo P, et al. (2003). Substitution of nevirapine, efavirenz, or abacavir for protease inhibitors in patients with human immunodeficiency virus infection. N Engl J Med. 349: 1036-1046.

[31] Walli R, Herfort O. Michl GM, Demant T, Jager H, Dieterle C, et al. (1998). Treatment with protease inhibitors associated with peripheral insulin resistance and impaired oral glucose tolerance in HIV-1-infected patients. AIDS. 2: F167-F173.

[32] Periard D, Telenti A, Sudre P, Cheseaux JJ, Halfon P, Reymond MJ, et al. (1999). Atherogenic dyslipidemia in HIV-infected individuals treated with protease inhibitors. The Swiss HIV Cohort Study. Circulation. 100: 700-705.

[33] DuPont Pharmaceuticals. Sustiva Prescribing Information. 2000.

[34] Glass TR, Ungsedhapand C, Wolbers M, Weber R, Vernazza PL, Rickenbach M, et al. (2006). Prevalence of risk factors for cardiovascular disease in HIV-infected patients over time: the Swiss HIV Cohort Study. HIV Med. 7: 404-410.

[35] Domingo P, Sambeat MA, Perez A, Ordonez J, Rodriguez J, Vazquez G. (2003). Fat distribution and metabolic abnormalities in HIV-infected patients on first combination antiretroviral therapy including stavudine or zidovudine: role of physical activity as a protective factor. Antivir Ther. 8: 223-231.

[36] van der Valk M, Kastelein JJ, Murphy RL, van Leth F, Katlama C, Horban A, et al. (2001). Nevirapine-containing antiretroviral therapy in HIV-1 infected patients results in an anti-atherogenic lipid profile. AIDS. 15: 2407-2414.

[37] Galli M, Ridolfo AL, Adorni F, Gervasoni C, Ravasio L, Corsico $\mathrm{L}$, et al. (2002). Body habitus changes and metabolic alterations in protease inhibitor-naive HIV-1-infected patients treated with two nucleoside reverse transcriptase inhibitors. J Acquir Immune Defic Syndr. 29: 21-31.

[38] Constans J, Pellegrin JL, Peuchant E, Dumon MF, Pellegrin I, Sergeant C, et al. (1994). Plasma lipids in HIV-infected patients: a prospective study in 95 patients. Eur J Clin Invest 24: 416-420.

[39] Vergis EN, Paterson DL, Wagener MM, Swindells S, Singh N. (2001). Dyslipidemia in HIV-infected patients: association with adherence to potent antiretroviral therapy. Int J STD AIDS. 12: 463-468.

[40] Lo JC, Kazemi MR, Hsue PY, Martin JN, Deeks SG, Schambelan M, Mulligan K. (2005). The relationship between nucleoside analogue treatment duration, insulin resistance, and fasting arterialized lactate level in patients with HIV infection. Clin Infect Dis. 41: 1335-1340.

[41] Sattler FR, Qian D, Louie S, Johnson D, Briggs W, DeQuattro V, Dube MP. (2001). Elevated blood pressure in subjects with lipodystrophy. AIDS. 15: 2001-2010.

[42] Thiebaut R, El-Sadr WM, Friis-Moller N, Rickenbach M, Reiss P, Monforte AD, et al. (2005). Predictors of hypertension and changes of blood pressure in HIV-infected patients. Antivir Ther. 10: 811823.

[43] Grinspoon S, Carr A. (2005). Cardiovascular risk and body-fat abnormalities in HIV-infected adults. N Engl J Med. 352: 48-62

[44] Dube MP, Stein JH, Aberg JA, Fichtenbaum CJ, Gerber JG, Tashima KT, et al. (2003). Guidelines for the evaluation and management of dyslipidemia in human immunodeficiency virus (HIV)infected adults receiving antiretroviral therapy: recommendations of the HIV Medical Association of the Infectious Disease Society of America and the Adult AIDS Clinical Trials Group. Clin Infect Dis. 37: 613-627.

[45] Knobel H, Jericó C, Guelar A, Montero M, Sorli L, LópezColomés JL, Pedro-Botet J. (2006. Dislipemia asociada a lopinavir/ritonavir: impacto en el control desde una unidad específica. Enferm Infecc Microbiol Clin. 24 (Espec Congr): 133.

[46] Graham I, Atar D, Borch-Johnsen K, Boysen G, Burell G, Cifkova $\mathrm{R}$, et al. (2007). European guidelines on cardiovascular disease prevention in clinical practice: Fourth Joint Task Force of the European Society of Cardiology and other societies on cardiovascular disease prevention in clinical practice (constituted by representatives of nine societies and by invited experts). Eur J Cardiovasc Prev Rehabil. 14 Suppl 2: S1-113.

[47] Friis-Møller N, Weber R, D’Arminio Monforte A, El-Sadr W, Reiss $\mathrm{P}$, Dabis F, Morfeldt L, et al. Exposure to HAART Is Associated with an Increased Risk of Myocardial Infarction: The D:A:D Study: 2006, $13^{\text {th }}$ Conference on Retroviruses and Opportunistic Infections. Denver, USA p. 127.

[48] Law MG, Friis-Møller N, El-Sadr WM, Weber R, Reiss P, D'Arminio Monforte A, et al. (2006). The use of the Framingham equation to predict myocardial infarctions in HIV-infected patients: comparison with observed events in the D:A:D Study. HIV Med. 7: 218. 